\title{
A meta-analysis on the prevalence, associated factors and diagnostic methods of mental stress induced myocardial ischemia
}

\author{
Lijun Zhang ${ }^{1}$, Yanping Bao ${ }^{2 *}$, Xi Wang ${ }^{1}$, Yuxin Zhou ${ }^{3}$, Shuhui Tao ${ }^{1,4}$, Wan Xu ${ }^{1}$ and Meiyan Liu ${ }^{1 *}$
}

\begin{abstract}
Background: The high prevalence of mental stress induced myocardial ischemia (MSIMI) causes double risk of adverse cardiac events in patients with MSIMI. However, multiple types of mental stress, diagnostic techniques, and diagnostic measurements may increase the complexity and heterogeneity in the assessment of MSIMI. Therefore, we performed this meta-analysis to assess the prevalence, associated factors, and diagnostic methods of MSIMI.

Methods: We systematically searched PubMed, EMBACE, Web of Science, CNKI, Wanfang through 1 Feb 2020 in English and Chinese. Review Manager (RevMan) Version 5.3 and Stata 12.0 were used for data analyses.

Results: Twenty articles were enrolled. The pooled estimates for the prevalence of MSIMI in CAD patients was 32\%. Potential associated factors of MSIMI involved history of post myocardial infarction (MI), or coronary artery bypass graft (CABG) (RR: 1.29, 95\% Cl 1.00-1.66, P =0.05; RR: 1.59, 95\% Cl 1.00-2.52, P=0.05). Evidence supported that diagnostic methods could influence the prevalence of MSIMI. Significant differences of MSIMI prevalence were found in different types of mental stress (Public Speaking: 22\%; Mental arithmetic: 26\%; Anger recall: 34\%; Two types: 37\%; Three or more than three types: 43\%, P =0.02), diagnostic techniques (SPECT: 26\%; RNV: 38\%; ECG: 16\%; Echocardiography: 41\%; Two types: 43\%, P<0.0001), and diagnostic measurements (LVEF decrease: 19\%; WMA: 51\%; ST depression: 16\%; MPD: 26\%; Two or more than two measurements: 45\%, $\mathrm{P}<0.00001)$. Moreover, univariate meta-regression demonstrated that MSIMI was linked with mental stress (exp(b): 1.0508, SE: 0.0201, P: 0.018).

Conclusions: This meta-analysis implicated that patients with diabetes, post MI or CABG might be more vulnerable to MSIMI. However, the prevalence of MSIMI could be influenced by diagnostic methods, especially the adopted types of mental stress, diagnostic techniques and measurements. Therefore, it is necessary to formulate a standard diagnostic method for MSIMI, which should be adequate, assessable, and affordable worldwide.
\end{abstract}

Registration PROSPERO. Online Protocol: CRD42020162822.

Keywords: Mental stress, Myocardial ischemia, Diagnostic method, Meta-regression

*Correspondence: liumeiyanaz@ccmu.edu.cn; baoyp@bjmu.edu.cn ${ }^{1}$ Cardiology Department, Beijing Anzhen Hospital, Capital Medical University, No. 2 Anzhen Road, Chaoyang District, Beijing 100029, China ${ }^{2}$ National Institute on Drug Dependence, Peking University, No. 38, Xueyuan Road, Haidian District, Beijing 10091, China

Full list of author information is available at the end of the article

\section{Background}

Cardiovascular diseases (CVD) have been threatening human's life for a long time all around the world, which could lead to 23.3 million deaths by 2030 according to the report from World Health Organization [1]. In China, there are more than 290 million patients with established cardiovascular diseases, and the mortality keeps rising [2]. Tens of billions of dollars have been spent in CVD management with limited effect. Despite 
traditional risk factors such as smoke, hypertension, hyperlipidemia, and diabetes [3], increasing evidence identified mental stress as a crucial risk factor in the development and progression of CVD [4]. Researchers discovered that mental stress induced in the laboratory (e.g. mental arithmetic, public speaking, et al.) contributed to myocardial ischemia, which could be assessed by echocardiography, electrocardiogram, or SPECT (single photonemission computed tomography) [5-7]. The prevalence of mental stress induced myocardial ischemia (MSIMI) ranges from 50 to $70 \%$ in patients with coronary artery diseases (CAD) [8]. Compared with patients without MSIMI, patients with MSIMI have double risk of adverse cardiac events [8].

However, the mechanisms of MSIMI remain uncertain. Previous studies showed that emotional status such as the trait and state of anger [5], anxiety [6], depression [7] could have great impact. Potential mechanisms may involve inflammatory responses, cortisol responses, fibrinogen responses, coagulation system, hypothalamic pituitary adrenal (HPA) [9, 10]. Hammadah et al. [11] linked cardiac biomarker with MSIMI, presenting that patients with MSIMI had higher level of resting cTnI. However, various factors such as sex, race, disease history, and drug history, and multiple types of mental stress, diagnostic techniques, and diagnostic measurements increase the complexity and heterogeneity in the assessment of MSIMI. Therefore, we performed this meta-analysis and metaregression in an effort to explore the potential mediators of MSIMI.

\section{Methods}

\section{Article selection strategy}

This meta-analysis had been registered in PROSPERO (CRD42020162822). We conducted the present metaanalysis by searching PubMed, Embase, Web of science, China National Knowledge Infrastructure (CNKI), and Wanfang website through 1 Feb 2020, with key words "mental stress", "psychological stress", "myocardial ischemia", "mental stress ischemia", "mental stress induced myocardial ischemia", "MSIMI".

Inclusion criteria: (1) Prospective cohort study or cross-sectional study; (2) English or Chinese language; (3) Patients with coronary artery disease; (4) Full articles were able to be found; (5) The data were eligible to be extracted; (6) Articles with high or medium quality.

Exclusion criteria: (1) Articles with repeated data from the same study project; (2) Mental stress tasks followed exercise stress at the same day, which might implicated that myocardial ischemia could be induced by exercise stress rather than mental stress.

\section{Article selection steps}

Three authors focused on selecting the proper articles in nearly 1 month. There were four steps in article selection and data extraction. First, the authors read the titles and excluded those unsuitable; Second, they read the abstracts and included those articles in the scope of our research; Third, they downloaded the articles with full text through the internet or our country library; Fourth, they read all articles, extracted necessary data for this study, and excluded articles without qualified data. Agreement must be reached among three authors to process the data.

\section{Quality assessment}

The quality of the cross-sectional studies was assessed by Crombie tool [12]. According to the scores, the article was classified into Grade A (6.0-7.0 points), Grade B (4.0-5.5 points), Grade C ( $<4$ points). Articles with Grade A were regarded as high quality, Grade B as moderate quality, and Grade $C$ as low quality. The quality of the prospective cohort studies was assessed by Newcastle-Ottawa Scale (NOS) [13]. Articles with seven to nine stars were estimated as high quality, five to six stars as medium quality, and zero to four stars as poor quality. Articles with high or medium quality were included in the present study (Table 1).

\section{Data extraction}

The data were extracted by two researchers separately and reached agreement after consultation. The following data were extracted: first author; publication date; country; total sample size; the sample of patients with MSIMI; study type; mental stress; diagnostic methods; scores of Crombie/NOS; article quality. All the data were presented in Table 1.

\section{Diagnostic methods of MSIMI}

1. Mental stress: Participants received one or more than one type of mental stress for $5 \mathrm{~min}$, involving the most common types: mental arithmetic, public speaking, mirror trace, Stroop color word task, and several other uncommon types of mental stress (such as reading).

2. Diagnostic techniques: Several techniques were adapted to evaluate cardiac function before and after participants went through mental stress, such as electrocardiogram (ECG), echocardiography, single photon emission computed tomography (SPECT), ventricular function monitor, radionuclide ventriculography (RNV).

3. Diagnostic criteria: Researchers have developed 4 criteria to diagnose MSIMI, including left ventricular 


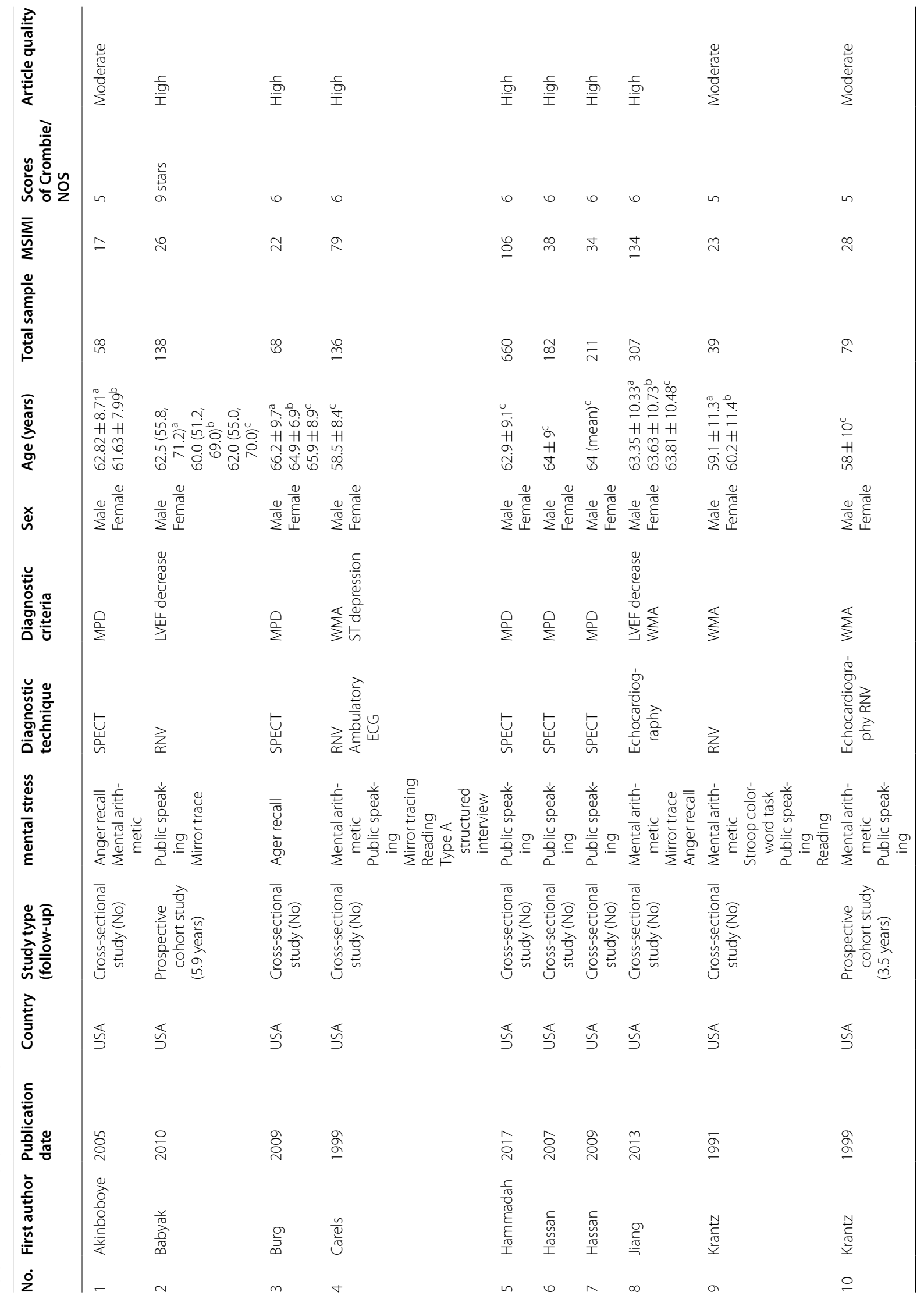




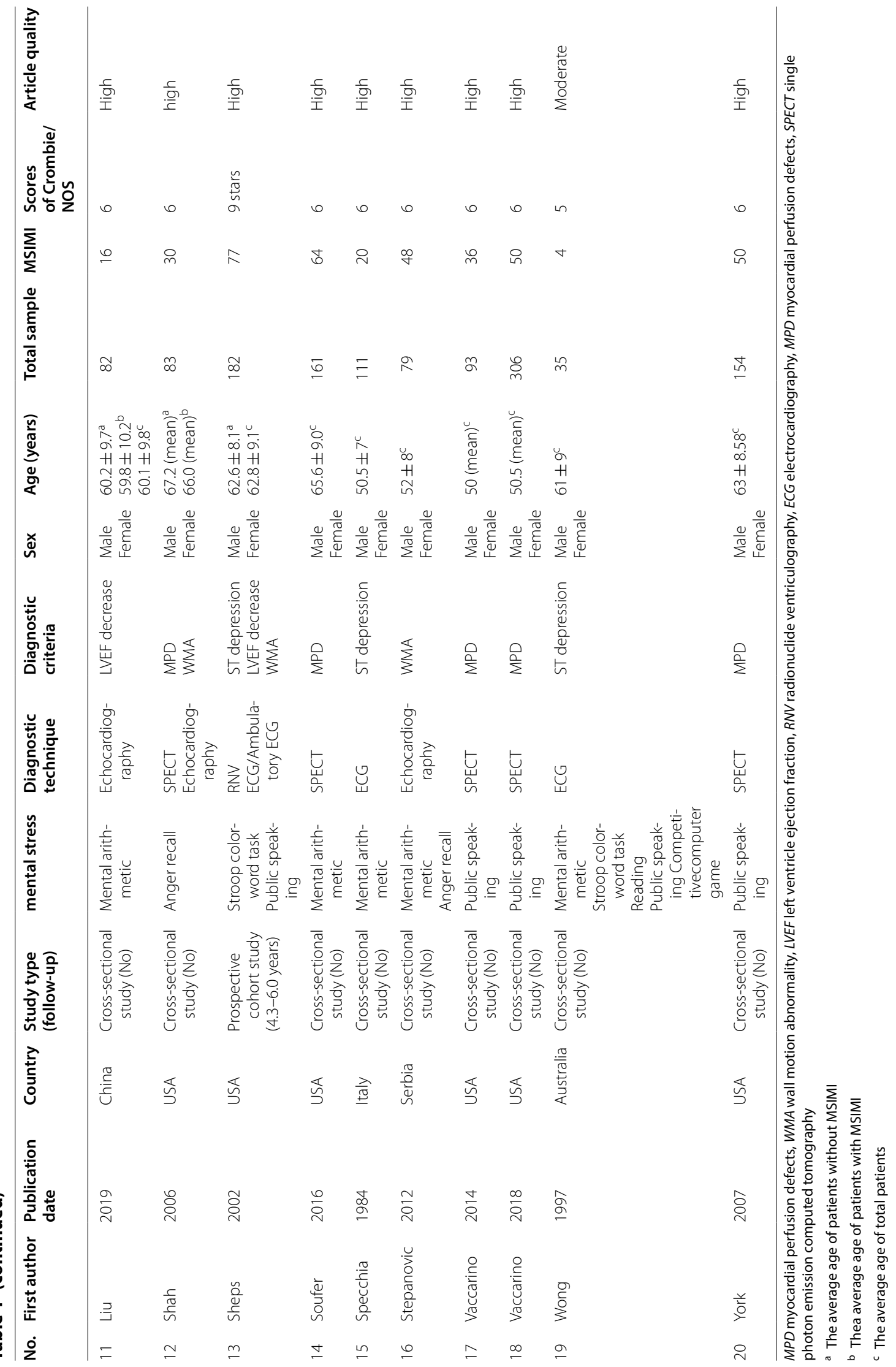


ejection fraction (LVEF) decrease $\geq 5 \%$ or $8 \%$, new or worsen wall motion abnormality, myocardial perfusion defect, ST depression $\geq 0.1 \mathrm{mV}$. Any of the four criteria could be adequate to diagnose MSIMI.

More details about diagnostic methods were shown in Table 2.

\section{Statistical analysis}

Review Manager (RevMan) Version 5.3 and Stata 12.0 were adopted for data analyses. Cochran's Q-test [14] and $\mathrm{I}^{2}$ statistic [15] were used for heterogeneity. Pooled effect size was analyzed by random-effects model or fixed-effects model according to the level of heterogeneity. Random-effects model was established for significant heterogeneity $\left(\mathrm{P}<0.10\right.$ or $\left.\mathrm{I}^{2}>50 \%\right)$, while fixed-effects model was used for non-significant heterogeneity
( $\mathrm{P}>0.10$ or $\left.\mathrm{I}^{2}<50 \%\right)$. Meta-Regression and subgroup analysis were applied for seeking heterogeneity sources. Sensitivity analyses were performed via excluding studies one at a time [16]. Publication bias was estimated by funnel plot and Begg' test [17]. P values were two-sided, and $\mathrm{P}<0.05$ was considered statistically significant.

\section{Results}

Prevalence of MSIMI in patients with CAD

For this meta-analysis, a total number of 30,080 publications were found from the databases. After removing duplication and articles unrelated to the topic, 20 eligible articles were finally selected [18-37]. Sixteen studies came from USA, and other four came from China, Serbia, Italy, Australia respectively. The flow chart was presented in Fig. 1. This meta-analysis enrolled 3164 patients with CAD, including 902 patients with MSIMI,

Table 2 The details of diagnostic methods

\begin{tabular}{|c|c|c|}
\hline Diagnostic methods & Types & Details \\
\hline \multirow[t]{8}{*}{ Mental stress } & Mental arithmetic & $\begin{array}{l}\text { Participants were required to complete a series of mathematical calculation, for instance, } \\
\text { to subtract } 7 \text { from a 4-digit number in } 5 \text { min as quickly as possible, at the same time, they } \\
\text { would receive encouragement or discouragement from the investigators }\end{array}$ \\
\hline & Public speaking & $\begin{array}{l}\text { Participants were asked to give a speech on a topic given by the investigators, and they had } \\
2 \text { min to prepare and } 3 \text { min to deliver the speech. They were told that their speech would } \\
\text { be recorded and evaluated by the investigators }\end{array}$ \\
\hline & Mirror trace & Participants were instructed to outline the shape of a star from its reflection in a mirror \\
\hline & Stroop color word task & $\begin{array}{l}\text { Participants were showing a series of slides which displaying the written word of a non- } \\
\text { matching color (e.g. the word green in blue color) }\end{array}$ \\
\hline & Anger recall & $\begin{array}{l}\text { Participants were asked to recall a recent annoying event which made them feel angry, } \\
\text { upset, irritated, frustrated, then described the situation and feeling to the investigators in } \\
\text { details }\end{array}$ \\
\hline & Reading & $\begin{array}{l}\text { Participants were asked to read a passage given by the investigators, such as neutral pas- } \\
\text { sage, in front of the investigators }\end{array}$ \\
\hline & Type A structured interview & $\begin{array}{l}\text { Participants underwent a standard videotaped interview to assess type A behavior which } \\
\text { might last } 20 \text { min }\end{array}$ \\
\hline & Competitive computer game & $\begin{array}{l}\text { Participants were asked to play a kind of computer game, which might elicit threat, uncer- } \\
\text { tainty, and avoidance. }\end{array}$ \\
\hline \multirow[t]{4}{*}{ Diagnostic techniques } & SPECT & $\begin{array}{l}\text { [99mTc] sestamibi SPECT was used to acquire myocardial perfusion imaging at rest and } \\
\text { during mental stress }\end{array}$ \\
\hline & RNV & $\begin{array}{l}\text { R-wave synchronized, multiple-gated RNV was conducted with a gamma camera posi- } \\
\text { tioned in the left anterior oblique angle, to acquire LVEF and left ventricular wall motion }\end{array}$ \\
\hline & ECG/Ambulatory ECG & 12 lead ECG or an ambulatory ECG was used for recording ST segments \\
\hline & Echocardiography & Two dimensional echocardiography was used to assess regional wall motion and LVEF \\
\hline \multirow[t]{4}{*}{ Diagnostic measurements } & LVEF decrease & $\begin{array}{l}\text { A reduction of LVEF at least } 5 \% \text { or } 8 \% \text { during mental stress compared with rest LVEF was } \\
\text { considered to exhibit MSIMI }\end{array}$ \\
\hline & WMA & New or worsened wall motion abnormalities during mental stress when compared with rest \\
\hline & ST depression & At least 1 mm ST segment depression by ECG or ambulatory ECG \\
\hline & MPD & $\begin{array}{l}\text { A 17-segment model was used to assess the myocardial perfusion defects comparing rest } \\
\text { and mental stress images, The following considerations could be regarded as MSIMl: a } \\
\text { new myocardial perfusion defect with a score of } 2 \text { in any segment, or worsening of a } \\
\text { preexisting impairment of at least } 2 \text { points in a single segment, or worsening of at least } 1 \\
\text { point in } 2 \text { or more contiguous segments }\end{array}$ \\
\hline
\end{tabular}




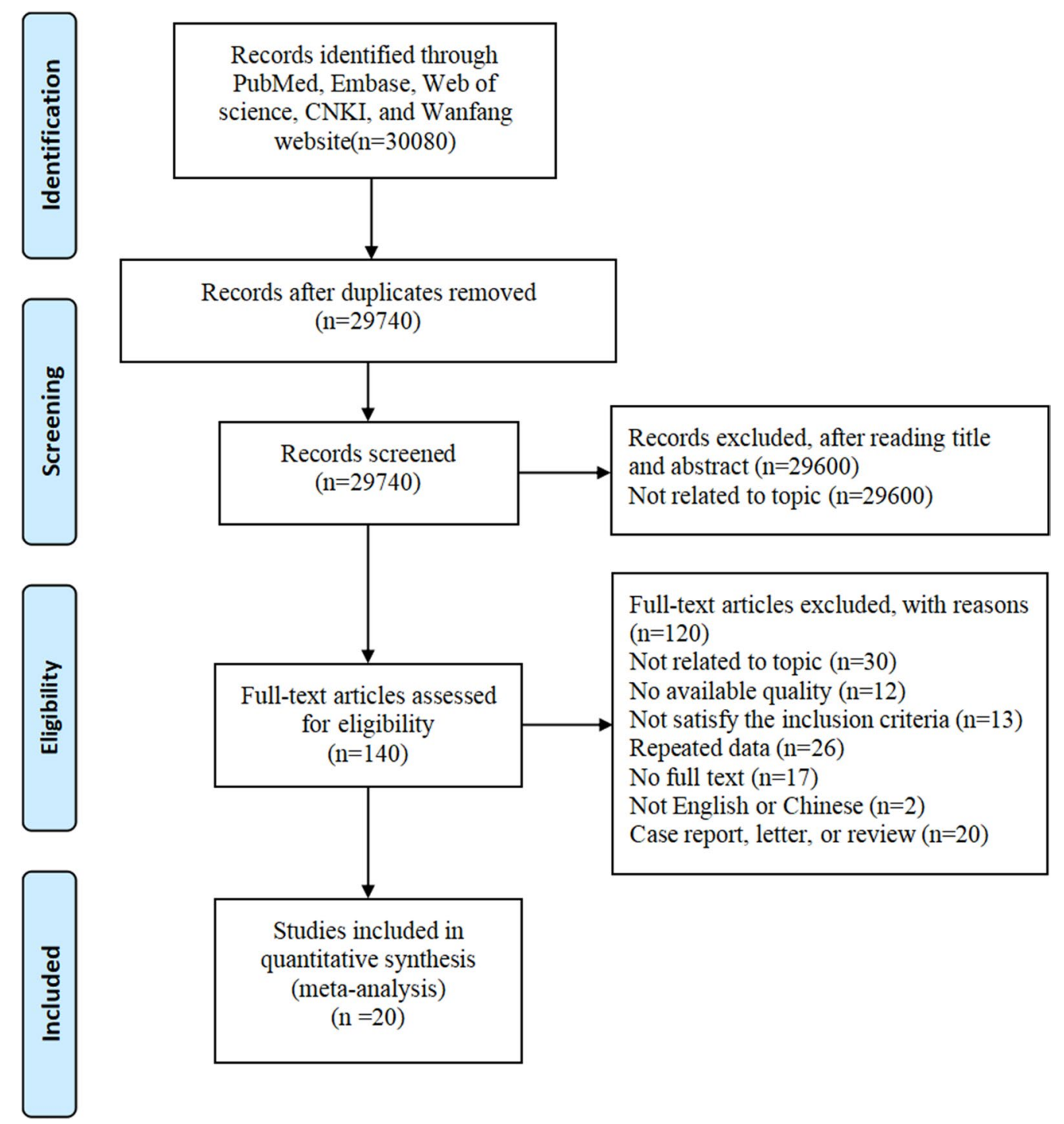

Fig. 1 The flow diagram of meta-analysis on mental stress induced myocardial ischemia; CNKI: China National Knowledge Infrastructure

and 2262 patients without MSIMI. The characteristics of all the articles were presented in Table 1 (Fig. 1, Table 1).

Of the 20 studies, the prevalence of MSIMI in CAD patients ranges from 11 to $61 \%$. In this meta-analysis, the pooled estimate for the prevalence of MSIMI in CAD patients is $32 \%$ (95\% CI 0.26, 0.38) (Fig. 2). We performed subgroup analyses of MSIMI prevalence, according to sex (Female 30\%, Male 31\%), race (White 40\%, non-white 47\%), smoking (Smoke+34\%, Smoke-31\%), disease history (Hypertension $+34 \%$, Hypertension-30\%, Hyperlipidemia + 36\%, Hyperlipidemia-29\%, Diabetes+38\%, Diabetes-31\%, Depression $+56 \%$, Depression $-31 \%$, Post MI $+38 \%$, Post MI $-32 \%$, PTCA $+32 \%$, PTCA-34\%, CABG $+37 \%$,
CABG-30\%), and drug history (Aspirin+33\%, Aspirin- $32 \%$, Other antiplatelets $+33 \%$, Other antiplatelets $-32 \%, \quad \mathrm{ACEI}+34 \%, \mathrm{ACEI}-33 \%, \mathrm{ARB}+35 \%$, ARB- $29 \%, \beta$-block $+31 \%, \beta$-block-30\%, CCB $+32 \%$, CCB $-34 \%$, Statins $+31 \%$, Statins $-19 \%$ ) (Table 3).

\section{Potential associated factors of MSIMI History of post MI}

Five articles [19, 22, 25, 31, 33] were selected in the subgroup comparison of post MI history, including 585 patients with post MI and 760 without. Difference of MSIMI was found between patients with post MI and patients without (RR: $1.29,95 \%$ CI $1.00-1.66, \mathrm{P}=0.05$ ). 


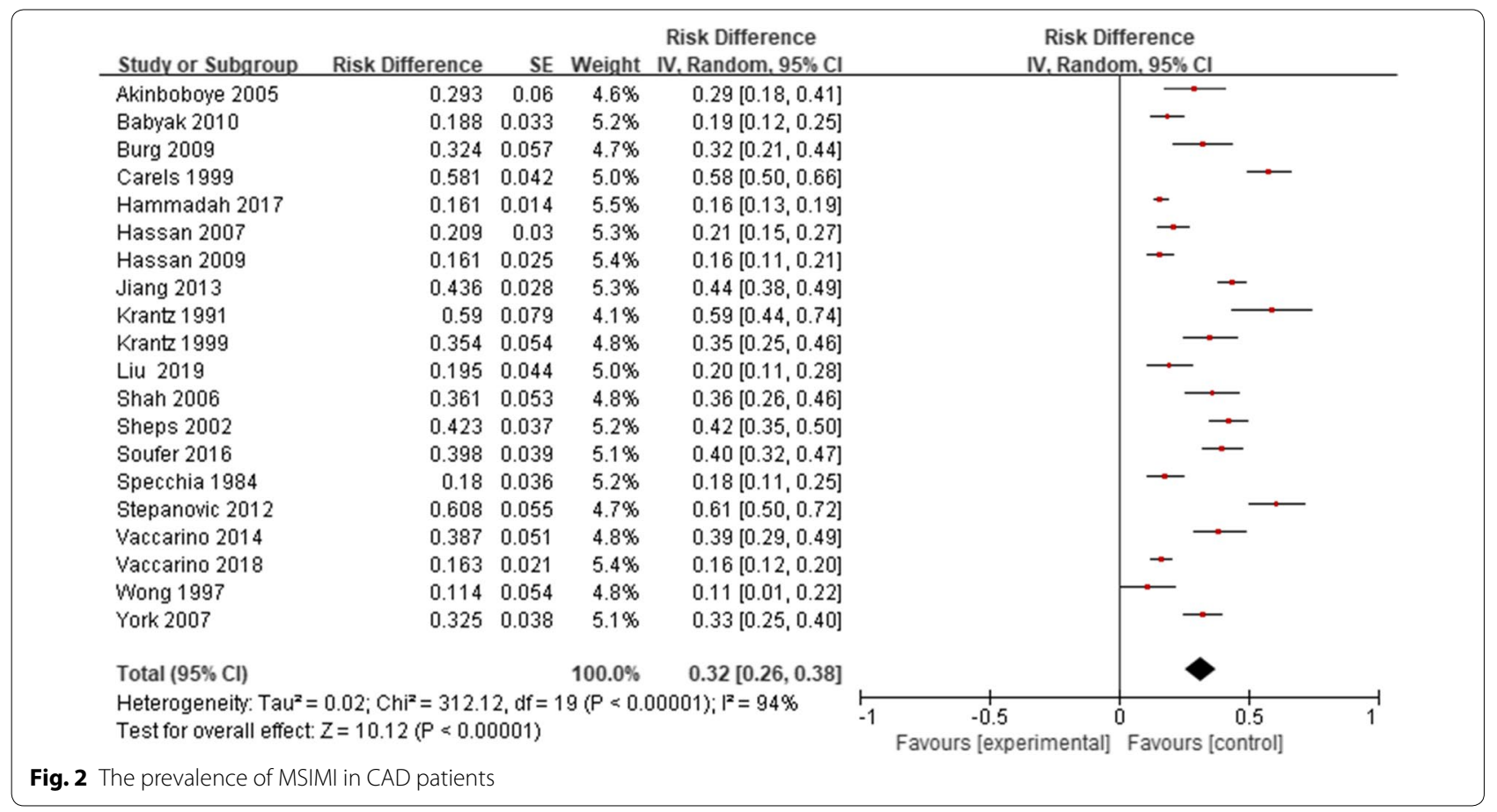

This result indicated that patients with post MI history might be at higher risk of MSIMI (Fig. 3a, Table 4).

\section{History of $C A B G$}

Three articles $[22,25,42]$ were selected in the subgroup comparison of history of CABG, including 432 patients with CABG history and 696 patients without. Difference of MSIMI was found between patients with CABG and patients without (RR: $1.59,95 \%$ CI 1.00-2.52, $\mathrm{P}=0.05$ ), indicating that patients with history of CABG might be at higher risk of developing MSIMI (Fig. 3b, Table 4).

\section{History of diabetes}

Eight articles [19, 20, 22, 25, 28, 29, 31, 33] were selected in the subgroup comparison of history of diabetes, including 608 patients with diabetes history and 1416 patients without. Although no significant difference was found (RR: $1.26,95 \%$ CI $0.98-1.62, \mathrm{P}=0.07$ ), we still considered the potential risk of diabetes in MSIMI due to its impact in coronary artery disease.

\section{Other characteristics}

Other characteristics were also conducted in this metaanalysis including sex (RR: 1.17, 95\% CI 0.93-1.48, $\mathrm{P}=0.18$ ), race (RR: $0.75,95 \% \mathrm{CI} 0.48-1.17, \mathrm{P}=0.21$ ), smoking (RR: $1.10,95 \%$ CI $0.86-1.40, \mathrm{P}=0.47$ ), hypertension (RR: $1.07,95 \%$ CI $0.80-1.42, \mathrm{P}=0.66$ ), hyperlipidemia (RR: 1.13, 95\% CI 0.80-1.60, P=0.48), PTCA (RR: $0.88,95 \%$ CI $0.67-1.16, P=0.37$ ), depression (RR: 1.36 ,
95\% CI 0.78-2.39, $\mathrm{P}=0.28)$, drug history such as aspirin (RR: $0.93,95 \%$ CI $0.65-1.34, \mathrm{P}=0.72$ ), other antiplatelets (RR: $1.21,95 \%$ CI 0.91-1.61, $\mathrm{P}=0.20)$, ACEI (RR: 1.13, 95\% CI 0.87-1.46, P=0.36), ARB (RR: $1.22,95 \%$ CI 0.53-2.82, $\mathrm{P}=0.64$ ), $\beta$-block (RR: $1.05,95 \%$ CI 0.78-1.41, $\mathrm{P}=0.75$ ), CCB (RR: $0.84,95 \%$ CI $0.58-1.22, \mathrm{P}=0.36$ ), statins (RR: $1.18,95 \% \mathrm{CI} 0.80-1.75, \mathrm{P}=0.40$ ), and no significant difference was found (Table 4).

\section{Diagnostic methods of MSIMI}

There were significant differences in the prevalence of MSIMI in different types of mental stress, diagnostic techniques, and diagnostic measurements. The prevalence of MSIMI detected by Public Speaking was 22\%, Mental arithmetic was 26\%, Anger recall was 34\%, Two types was $37 \%$, three or more than three types was $43 \%$, and the result was significant $(P=0.02)$. The results indicated that two and more than two types of mental stress could be more likely to induce MSIMI. The prevalence of MSIMI detected by different types of diagnostic techniques and diagnostic measurements showed significant difference (Table 3, Figs. 4, 5, 6).

Meta-regression was performed to identify the potential moderators in the prevalence of MSIMI, including publication date, sample size, country, different types of mental stress, different types of diagnostic techniques, and different types of myocardial ischemia measurements (Tables 3 and 5). 
Table 3 Prevalence of subgroups analyses

\begin{tabular}{|c|c|c|c|c|c|c|c|c|}
\hline Subgroups & No. of studies & Total & MSIMI & $\begin{array}{l}\text { Pooled } \\
\text { prevalence(\%) }\end{array}$ & $95 \% \mathrm{Cl}$ & Effect Model & Heterogeneity & $\begin{array}{l}\text { Test for } \\
\text { overall effect }\end{array}$ \\
\hline \multicolumn{9}{|l|}{ Basic characteristics } \\
\hline Female & 11 & 612 & 162 & 30 & $0.21,0.39$ & Random & $\begin{array}{l}\mathrm{Tau}^{2}=0.02 ; \\
\mathrm{Chi}^{2}=55.51, \mathrm{df}=10 \\
(P<0.00001) \\
\mathrm{I}^{2}=82 \%\end{array}$ & $Z=6.66(P<0.00001)$ \\
\hline Male & 11 & 1519 & 420 & 31 & $0.22,0.40$ & Random & $\begin{array}{l}\mathrm{Tau}^{2}=0.02 \\
\mathrm{Chi}^{2}=148.60 \\
\mathrm{df}=10(\mathrm{P}<0.00001) \\
\mathrm{I}^{2}=93 \%\end{array}$ & $Z=7.07(P<0.00001)$ \\
\hline White & 3 & 440 & 175 & 40 & $0.35,0.44$ & Fix & $\begin{array}{l}C h i^{2}=1.37, d f=2 \\
(P=0.50) ; l^{2}=0 \%\end{array}$ & $Z=17.02(P<0.00001)$ \\
\hline Other races & 3 & 96 & 45 & 47 & $0.37,0.57$ & Fix & $\begin{array}{l}\mathrm{Chi}^{2}=2.94, \mathrm{df}=2 \\
(P=0.23) ; I^{2}=32 \%\end{array}$ & $Z=0.54(P=0.59)$ \\
\hline Smoke+ & 8 & 902 & 256 & 34 & $0.21,0.46$ & Random & $\begin{array}{l}\mathrm{Tau}^{2}=0.03 \\
\mathrm{Chi}^{2}=103.54 \\
\mathrm{df}=7(\mathrm{P}<0.00001) \\
\mathrm{I}^{2}=93 \%\end{array}$ & $Z=5.43(P<0.00001)$ \\
\hline Smoke- & 8 & 676 & 190 & 31 & $0.20,0.42$ & Random & $\begin{array}{l}\mathrm{Tau}^{2}=0.02 \\
\mathrm{Chi}^{2}=65.09, \mathrm{df}=7 \\
(P<0.00001) \\
\mathrm{I}^{2}=89 \%\end{array}$ & $Z=5.48(P<0.00001)$ \\
\hline Hypertension+ & 8 & 1211 & 355 & 34 & $0.23,0.44$ & Random & $\begin{array}{l}\mathrm{Tau}^{2}=0.02 \\
\mathrm{Chi}^{2}=102.11 \\
\mathrm{df}=7(\mathrm{P}<0.00001) \\
\mathrm{I}^{2}=93 \%\end{array}$ & $Z=6.25(P<0.00001)$ \\
\hline Hypertension- & 8 & 367 & 91 & 30 & $0.18,0.43$ & Random & $\begin{array}{l}\mathrm{Tau}^{2}=0.03 \\
\mathrm{Chi}^{2}=52.54, \mathrm{df}=7 \\
(P<0.00001) \\
\mathrm{I}^{2}=87 \%\end{array}$ & $Z=4.72(P<0.00001)$ \\
\hline Hyperlipidemia+ & 8 & 1255 & 381 & 36 & $0.24,0.47$ & Random & $\begin{array}{l}\mathrm{Tau}^{2}=0.03 \\
\mathrm{Chi}^{2}=135.94 \\
\mathrm{df}=7(\mathrm{P}<0.00001) \\
\mathrm{I}^{2}=95 \%\end{array}$ & $Z=6.09(P<0.00001)$ \\
\hline Hyperlipidemia- & 8 & 185 & 39 & 29 & $0.17,0.40$ & Random & $\begin{array}{l}\mathrm{Tau}^{2}=0.01 \\
\quad C h i^{2}=15.92, \mathrm{df}=6 \\
(P=0.01) ; l^{2}=62 \%\end{array}$ & $Z=4.86(P<0.00001)$ \\
\hline Diabetes+ & 8 & 466 & 142 & 38 & $0.25,0.52$ & Random & $\begin{array}{l}\mathrm{Tau}^{2}=0.03 \\
\mathrm{Chi}^{2}=63.60, \mathrm{df}=7 \\
(P<0.00001) \\
\mathrm{I}^{2}=89 \%\end{array}$ & $Z=5.54(P<0.00001)$ \\
\hline Diabetes- & 8 & 1112 & 304 & 31 & $0.20,0.42$ & Random & $\begin{array}{l}\mathrm{Ta}^{2}=0.02 \\
\mathrm{Chi}^{2}=113.93 \\
\mathrm{df}=7(\mathrm{P}<0.00001) \\
\mathrm{I}^{2}=94 \%\end{array}$ & $Z=5.58(P<0.00001)$ \\
\hline Depression+ & 2 & 71 & 28 & 56 & $0.15,2.08$ & Random & $\begin{array}{l}\mathrm{Tau}^{2}=0.75 \\
\mathrm{Chi}^{2}=5.93, \mathrm{df}=1 \\
(P=0.01) ; I^{2}=83 \%\end{array}$ & $Z=0.87(P=0.39)$ \\
\hline Depression- & 2 & 318 & 122 & 31 & $0.07,0.54$ & Random & $\begin{array}{l}\mathrm{Tau}^{2}=0.03 \\
\mathrm{Chi}^{2}=15.40, \mathrm{df}=1 \\
(\mathrm{P}<0.0001) ; I^{2}=94 \%\end{array}$ & $Z=2.58(P=0.010)$ \\
\hline Post Ml+ & 5 & 585 & 189 & 38 & $0.21,0.55$ & Random & $\begin{array}{l}\mathrm{Tau}^{2}=0.03 \\
\mathrm{Chi}^{2}=78.29, \mathrm{df}=4 \\
(P<0.00001) \\
\mathrm{I}^{2}=95 \%\end{array}$ & $Z=4.43(P<0.00001)$ \\
\hline Post Ml- & 5 & 760 & 189 & 32 & $0.17,0.46$ & Random & $\begin{array}{l}\mathrm{Tau}^{2}=0.02 \\
C \mathrm{Ch}^{2}=62.36, \mathrm{df}=4 \\
(P<0.00001) \\
\mathrm{I}^{2}=94 \%\end{array}$ & $Z=4.36(P<0.0001)$ \\
\hline
\end{tabular}


Table 3 (continued)

\begin{tabular}{|c|c|c|c|c|c|c|c|c|}
\hline Subgroups & No. of studies & Total & MSIMI & $\begin{array}{l}\text { Pooled } \\
\text { prevalence(\%) }\end{array}$ & $95 \% \mathrm{Cl}$ & Effect Model & Heterogeneity & $\begin{array}{l}\text { Test for } \\
\text { overall effect }\end{array}$ \\
\hline PTCA+ & 3 & 633 & 168 & 32 & $0.11,0.53$ & Random & $\begin{array}{l}\mathrm{Tau}^{2}=0.03 \\
\mathrm{Chi}^{2}=59.22, \mathrm{df}=2 \\
(\mathrm{P}<0.00001) \\
\mathrm{I}^{2}=97 \%\end{array}$ & $Z=2.97(P=0.003)$ \\
\hline PTCA- & 3 & 495 & 136 & 34 & $0.14,0.54$ & Random & $\begin{array}{l}\mathrm{Tau}^{2}=0.03 \\
\quad C h i 2=37.29, \mathrm{df}=2 \\
(P<0.00001) \\
I^{2}=95 \%\end{array}$ & $Z=3.40(P=0.0007)$ \\
\hline CABG+ & 3 & 432 & 148 & 37 & $0.21,0.54$ & Random & $\begin{array}{l}\mathrm{Tau}^{2}=0.02 \\
\mathrm{Chi}^{2}=22.92, \mathrm{df}=2 \\
(\mathrm{P}<0.00001) \\
\mathrm{I}^{2}=91 \%\end{array}$ & $Z=4.57(P<0.00001)$ \\
\hline CABG- & 3 & 696 & 156 & 30 & $0.08,0.52$ & Random & $\begin{array}{l}\mathrm{Tau}^{2}=0.04 ; \\
\mathrm{Chi}^{2}=70.62, \mathrm{df}=2 \\
(P<0.00001) \\
\mathrm{I}^{2}=97 \%\end{array}$ & $Z=2.67(P=0.008)$ \\
\hline Aspirin+ & 5 & 1081 & 298 & 33 & $0.18,0.48$ & Random & $\begin{array}{l}\mathrm{Tau}^{2}=0.03 \\
\mathrm{Chi}^{2}=91.34, \mathrm{df}=4 \\
(P<0.00001) \\
\mathrm{I}^{2}=96 \%\end{array}$ & $Z=4.34(P<0.0001)$ \\
\hline Aspirin- & 5 & 198 & 58 & 32 & $0.20,0.45$ & Random & $\begin{array}{l}\mathrm{Tau}^{2}=0.01 \\
\mathrm{Chi}^{2}=12.74, \mathrm{df}=4 \\
(P=0.01) ; l^{2}=69 \%\end{array}$ & $Z=5.08(P<0.00001)$ \\
\hline Other antiplatelets+ & 3 & 396 & 117 & 33 & $0.11,0.55$ & Random & $\begin{array}{l}\mathrm{Tau}^{2}=0.04 ; \\
\mathrm{Chi}^{2}=38.94, \mathrm{df}=2 \\
(\mathrm{P}<0.00001) \\
\mathrm{I}^{2}=95 \%\end{array}$ & $Z=2.96(P=0.003)$ \\
\hline Other antiplatelets- & 3 & 732 & 187 & 32 & $0.12,0.51$ & Random & $\begin{array}{l}\mathrm{Tau}^{2}=0.03 \\
\mathrm{Chi}^{2}=59.37, \mathrm{df}=2 \\
(\mathrm{P}<0.00001) \\
\mathrm{I}^{2}=97 \%\end{array}$ & $Z=3.19(P=0.001)$ \\
\hline ACEI+ & 5 & 658 & 201 & 34 & $0.21,0.47$ & Random & $\begin{array}{l}\mathrm{Tau}^{2}=0.02 ; \\
\mathrm{Chi}^{2}=42.57, \mathrm{df}=4 \\
(P<0.00001) \\
\mathrm{I}^{2}=91 \%\end{array}$ & $Z=5.24(P<0.00001)$ \\
\hline ACEI- & 5 & 621 & 155 & 33 & $0.17,0.49$ & Random & $\begin{array}{l}\mathrm{Tau}^{2}=0.03 \\
\mathrm{Chi}^{2}=58.21, \mathrm{df}=4 \\
(\mathrm{P}<0.00001) \\
\mathrm{I}^{2}=93 \%\end{array}$ & $Z=4.03(P<0.0001)$ \\
\hline ARB+ & 2 & 149 & 38 & 35 & $-0.08,0.78$ & Random & $\begin{array}{l}\mathrm{Tau}^{2}=0.09 \\
\quad \mathrm{Chi}^{2}=26.62, \mathrm{df}=1 \\
(\mathrm{P}<0.00001) \\
\mathrm{I}^{2}=96 \%\end{array}$ & $Z=1.61(P=0.11)$ \\
\hline ARB- & 2 & 818 & 202 & 29 & $0.04,0.54$ & Random & $\begin{array}{l}\mathrm{Tau}^{2}=0.03 \\
\mathrm{Chi}^{2}=54.50, \mathrm{df}=1 \\
(\mathrm{P}<0.00001) \\
\mathrm{I}^{2}=98 \%\end{array}$ & $Z=2.30(P=0.02)$ \\
\hline$\beta$-block+ & 6 & 1086 & 301 & 31 & $0.19,0.42$ & Random & $\begin{array}{l}\mathrm{Tau}^{2}=0.02 ; \\
\quad \mathrm{Chi}^{2}=85.31, \mathrm{df}=5 \\
(\mathrm{P}<0.00001) \\
\mathrm{I}^{2}=94 \%\end{array}$ & $Z=5.01(P<0.00001)$ \\
\hline$\beta$-block- & 6 & 331 & 81 & 30 & $0.19,0.41$ & Random & $\begin{array}{l}\mathrm{Tau}^{2}=0.01 ; \\
\mathrm{Chi}^{2}=20.32, \mathrm{df}=5 \\
(P=0.001) ;\left.\right|^{2}=75 \%\end{array}$ & $Z=5.31(P<0.00001)$ \\
\hline CCB+ & 4 & 165 & 56 & 32 & $0.20,0.43$ & Random & $\begin{array}{l}\mathrm{Tau}^{2}=0.01 \\
\mathrm{Chi}^{2}=7.26, \mathrm{df}=3 \\
(P=0.06) ; 1^{2}=59 \%\end{array}$ & $Z=5.49(P<0.00001)$ \\
\hline
\end{tabular}


Table 3 (continued)

\begin{tabular}{|c|c|c|c|c|c|c|c|c|}
\hline Subgroups & No. of studies & Total & MSIMI & $\begin{array}{l}\text { Pooled } \\
\text { prevalence(\%) }\end{array}$ & $95 \% \mathrm{Cl}$ & Effect Model & Heterogeneity & $\begin{array}{l}\text { Test for } \\
\text { overall effect }\end{array}$ \\
\hline CCB- & 4 & 509 & 190 & 34 & $0.21,0.47$ & Random & $\begin{array}{l}\mathrm{Tau}^{2}=0.02 ; \\
\mathrm{Chi}^{2}=28.66, \mathrm{df}=3 \\
(\mathrm{P}<0.00001) \\
\mathrm{I}^{2}=90 \%\end{array}$ & $Z=5.16(P<0.00001)$ \\
\hline Statins+ & 6 & 1236 & 344 & 31 & $0.19,0.43$ & Random & $\begin{array}{l}\mathrm{Tau}^{2}=0.02 ; \\
\mathrm{Chi}^{2}=96.14, \mathrm{df}=5 \\
(P<0.00001) \\
I^{2}=95 \%\end{array}$ & $Z=5.10(P<0.00001)$ \\
\hline Statins- & 6 & 181 & 38 & 19 & $0.14,0.25$ & Fix & $\begin{aligned} C h i^{2} & =8.00, d f=5 \\
(P & =0.16) ; I^{2}=38 \%\end{aligned}$ & $Z=6.77(P<0.00001)$ \\
\hline \multicolumn{9}{|l|}{ Country } \\
\hline USA & 16 & 2857 & 814 & 33 & $0.26,0.40$ & Random & $\begin{array}{l}\mathrm{Tau}^{2}=0.02 \\
\mathrm{Chi}^{2}=258.05 \\
\mathrm{df}=15(\mathrm{P}<0.00001) \\
\mathrm{I}^{2}=94 \%\end{array}$ & $Z=9.60(P<0.00001)$ \\
\hline \multirow[t]{2}{*}{ Other countries } & 4 & 307 & 88 & 27 & $0.08,0.46$ & Random & $\begin{array}{l}\operatorname{Tau}^{2}=0.04 \\
C h i^{2}=54.00, d f=3 \\
(P<0.00001) \\
I^{2}=94 \%\end{array}$ & $Z=2.78(P=0.005)$ \\
\hline & & & & & & & \multicolumn{2}{|c|}{$\begin{array}{l}\text { Test for subgroup differences: } \mathrm{Chi}^{2}=0.29 \\
\qquad \mathrm{df}=1(\mathrm{P}=0.59), \mathrm{I}^{2}=0 \%\end{array}$} \\
\hline \multicolumn{9}{|l|}{ Mental stress (MS) } \\
\hline Public speaking & 6 & 1606 & 314 & 22 & $0.17,0.28$ & Random & $\begin{array}{l}\operatorname{Tau}^{2}=0.00 ; \\
\mathrm{Chi}^{2}=34.85, \mathrm{df}=5 \\
(P<0.00001) \\
\mathrm{I}^{2}=86 \%\end{array}$ & $Z=7.95(P<0.00001)$ \\
\hline Mental arithmetic & 3 & 354 & 100 & 26 & $0.12,0.40$ & Random & $\begin{array}{l}\mathrm{Tau}^{2}=0.01 \\
\mathrm{Chi}^{2}=19.63, \mathrm{df}=2 \\
(P<0.0001) ; I^{2}=90 \%\end{array}$ & $Z=8.86(P<0.00001)$ \\
\hline Anger recall & 2 & 151 & 52 & 34 & $0.27,0.42$ & Fix & $\begin{array}{l}C h i^{2}=0.23, d f=1 \\
(P=0.63) ; l^{2}=0 \%\end{array}$ & \\
\hline Two MS & 5 & 536 & 196 & 37 & $0.23,0.51$ & Random & $\begin{array}{l}\mathrm{Tau}^{2}=0.02 \\
\mathrm{Chi}^{2}=50.67, \mathrm{df}=4 \\
(P<0.00001) \\
\mathrm{I}^{2}=92 \%\end{array}$ & $Z=5.12(P<0.00001)$ \\
\hline \multirow[t]{2}{*}{$\begin{array}{l}\text { Three or more than } \\
\text { three MS }\end{array}$} & 4 & 517 & 240 & 43 & $0.24,0.61$ & Random & $\begin{array}{l}\mathrm{Tau}^{2}=0.03 \\
\mathrm{Chi}^{2}=51.27, \mathrm{df}=3 \\
(P<0.00001) \\
\mathrm{I}^{2}=94 \%\end{array}$ & $Z=4.51(P<0.00001)$ \\
\hline & & & & & & & \multicolumn{2}{|c|}{$\begin{array}{l}\text { Test for subgroup differences: } \mathrm{Chi}^{2}=11.21 \\
\qquad \mathrm{df}=4(\mathrm{P}=0.02), \mathrm{I}^{2}=64.3 \%\end{array}$} \\
\hline \multicolumn{9}{|l|}{ Diagnostic techniques } \\
\hline SPECT & 9 & 1893 & 417 & 26 & $0.20,0.32$ & Random & $\begin{array}{l}\mathrm{Tau}^{2}=0.01 \\
\mathrm{Chi}^{2}=70.54, \mathrm{df}=8 \\
(P<0.00001) \\
\mathrm{I}^{2}=89 \%\end{array}$ & $Z=8.68(P<0.00001)$ \\
\hline RNV & 2 & 177 & 49 & 38 & $-0.01,0.78$ & Random & $\begin{array}{l}\mathrm{Tau}^{2}=0.08 \\
\mathrm{Chi}^{2}=22.05, \mathrm{df}=1 \\
(\mathrm{P}<0.00001) \\
\mathrm{I}^{2}=95 \%\end{array}$ & $\mathrm{Z}=1.90(\mathrm{P}=0.06)$ \\
\hline ECG & 2 & 146 & 24 & 16 & $0.10,0.22$ & Fix & $\begin{aligned} C h i^{2} & =1.03, d f=1 \\
(P & =0.31) ; I^{2}=3 \%\end{aligned}$ & $Z=5.33(P<0.00001)$ \\
\hline Echocardiography & 3 & 468 & 198 & 41 & $0.21,0.61$ & Random & $\begin{array}{l}\operatorname{Tau}^{2}=0.03 \\
\mathrm{Chi}^{2}=37.63, \mathrm{df}=2 \\
(P<0.00001) \\
\mathrm{I}^{2}=95 \%\end{array}$ & $Z=3.97(P<0.0001)$ \\
\hline $\begin{array}{l}\text { Two types of diag- } \\
\text { nostic technique }\end{array}$ & 4 & 480 & 214 & 43 & $0.33,0.54$ & Random & $\begin{array}{l}\mathrm{Tau}^{2}=0.01 \\
\quad \mathrm{Ch} \mathrm{i}^{2}=16.19, \mathrm{df}=3 \\
(P=0.001) ; 1^{2}=81 \%\end{array}$ & $Z=8.15(P<0.00001)$ \\
\hline
\end{tabular}


Table 3 (continued)

\begin{tabular}{|c|c|c|c|c|c|c|c|c|}
\hline Subgroups & No. of studies & Total & MSIMI & $\begin{array}{l}\text { Pooled } \\
\text { prevalence(\%) }\end{array}$ & $95 \% \mathrm{Cl}$ & Effect Model & Heterogeneity & $\begin{array}{l}\text { Test for } \\
\text { overall effect }\end{array}$ \\
\hline & & & & & & & \multicolumn{2}{|c|}{$\begin{array}{l}\text { Test for subgroup differences: } \mathrm{Chi}^{2}=23.61 \text {, } \\
\qquad \mathrm{df}=4(\mathrm{P}<0.0001), \mathrm{I}^{2}=83.1 \%\end{array}$} \\
\hline \multicolumn{9}{|c|}{ Myocardial ischemia measurements } \\
\hline LVEF decrease & 2 & 220 & 42 & 19 & $0.14,0.24$ & Fix & $\begin{array}{l}C h i^{2}=0.02, d f=1 \\
(P=0.90) ; l^{2}=0 \%\end{array}$ & $Z=7.22(P<0.00001)$ \\
\hline WMA & 3 & 197 & 99 & 51 & $0.34,0.69$ & Random & $\begin{array}{l}\mathrm{Tau}^{2}=0.02 \\
C \mathrm{Chi}^{2}=12.46, \mathrm{df}=2 \\
(P=0.002) ; I^{2}=84 \%\end{array}$ & $Z=5.78(P<0.00001)$ \\
\hline ST depression & 2 & 146 & 24 & 16 & $0.10,0.22$ & Fix & $\begin{array}{l}C h i^{2}=1.03, d f=1 \\
\quad(P=0.31) ; I^{2}=3 \%\end{array}$ & $Z=5.33(P<0.00001)$ \\
\hline MPD & 9 & 1893 & 417 & 26 & $0.20,0.32$ & Random & $\begin{array}{l}\mathrm{Tau}^{2}=0.01 \\
\mathrm{Chi}^{2}=70.54, \mathrm{df}=8 \\
(P<0.00001) \\
\mathrm{I}^{2}=89 \%\end{array}$ & $Z=8.68(P<0.00001)$ \\
\hline \multirow[t]{2}{*}{$\begin{array}{l}\text { Two or more than } \\
\text { two measure- } \\
\text { ments }\end{array}$} & 4 & 454 & 216 & 45 & $0.37,0.53$ & Random & $\begin{array}{l}\operatorname{Tau}^{2}=0.01 \\
\mathrm{Chi}^{2}=13.32, \mathrm{df}=3 \\
(\mathrm{P}=0.004) ; I^{2}=77 \%\end{array}$ & $Z=11.07(P<0.00001)$ \\
\hline & & & & & & & \multicolumn{2}{|c|}{$\begin{array}{l}\text { Test for subgroup differences: } \mathrm{Chi}^{2}=47.23 \\
\qquad \mathrm{df}=4(\mathrm{P}<0.00001), \mathrm{I}^{2}=91.5 \%\end{array}$} \\
\hline
\end{tabular}

MSIMI mental stress induced myocardial ischemia, $M I$ myocardial infarction, PTCA percutaneous coronary angioplasty, CABG coronary artery bypass graft, ACEI angiotensin converting enzyme inhibitor, $A R B$ angiotensin receptor block, $C C B$ calcium-channel blocker, $M S$ mental stress, SPECT single photon emission computed tomography, RNV radionuclide ventriculography, ECG electrocardiography, VEST ventricular function monitor, LVEF left ventricle ejection fraction, WMA wall motion abnormality, WMA wall motion abnormality, MPD myocardial perfusion defects

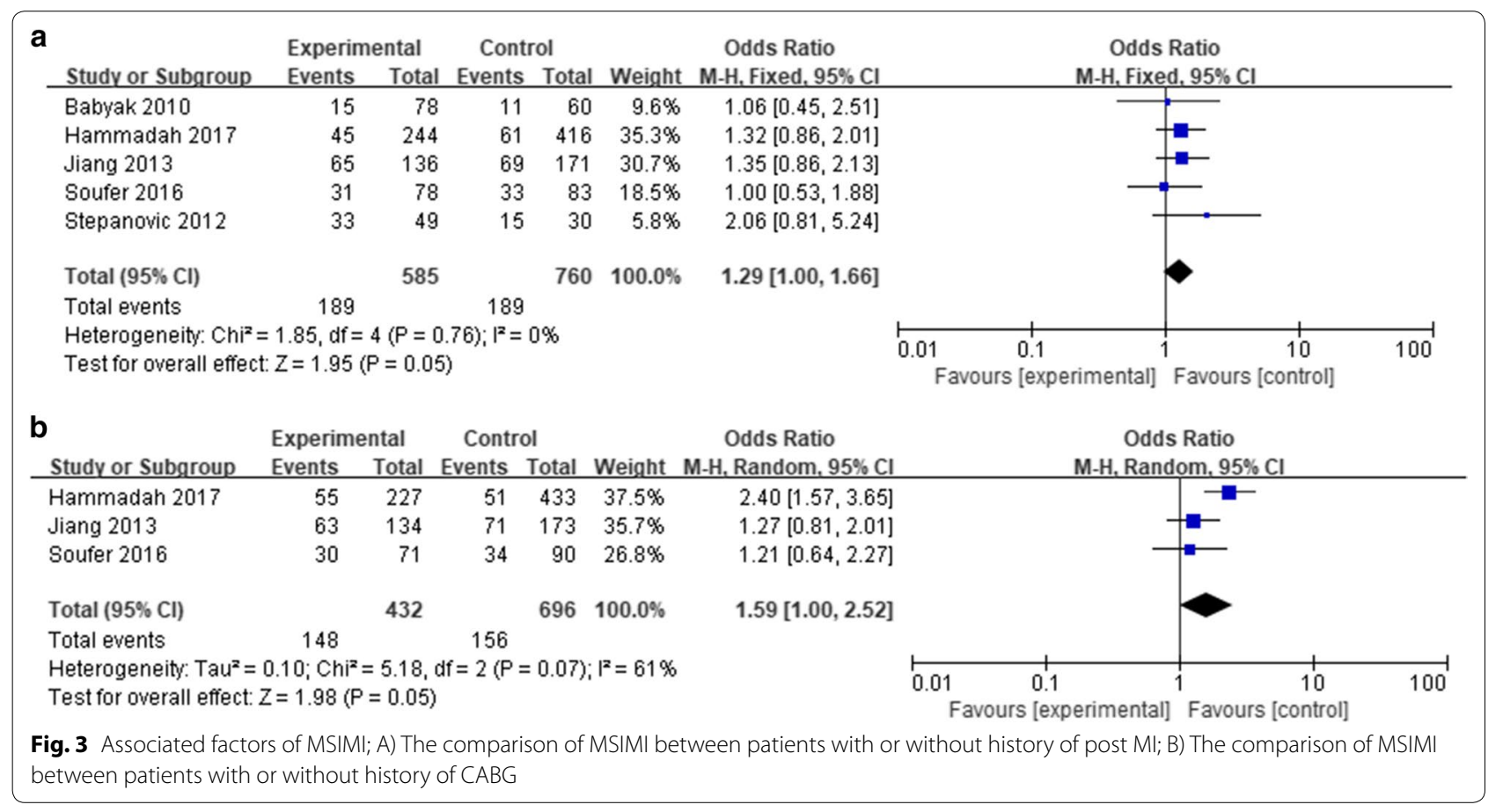

On univariate meta-regression, types of mental stress $(\exp (\mathrm{b}): 1.0508$, SE: 0.0201, P: 0.018) were associated with the prevalence of MSIMI, while no significance was found in other factors including publication year $(\exp (b)$ :
0.9977, SE: 0.0038, P: 0.549), sample size (exp(b): 0.9996, SE: 0.0002, P: 0.215), country (exp(b): 0.9433, SE: 0.0815, $\mathrm{P}:$ 0.508), diagnostic techniques $(\exp (\mathrm{b}): 1.0395$, SE: $0.0200, P: 0.060)$ and diagnostic measurements $(\exp (\mathrm{b})$ : 
Table 4 Subgroup comparisons results of the meta-analysis

\begin{tabular}{|c|c|c|c|c|c|c|c|}
\hline No. & Comparison & Studies (n) & Sample size & Effect Model & RR/MD/SMD & Heterogeneity & $\begin{array}{l}\text { Test for } \\
\text { overall effect }\end{array}$ \\
\hline 1 & Female vs. male & 11 & 2131 & Fix & $1.17[0.93,1.48]$ & $\begin{array}{l}\mathrm{Chi}^{2}=14.36, \mathrm{df}=10(\mathrm{P}=0.16) \\
\mathrm{I}^{2}=30 \%\end{array}$ & $Z=1.35(P=0.18)$ \\
\hline 2 & White vs. other races & 3 & 536 & Fix & $0.75[0.48,1.17]$ & $\begin{array}{l}C h i^{2}=1.32, d f=2(P=0.52) \\
\left.\right|^{2}=0 \%\end{array}$ & $Z=1.26(P=0.21)$ \\
\hline 3 & Smoke+vs. Smoke- & 8 & 1578 & Fix & $1.10[0.86,1.40]$ & $\begin{array}{l}\mathrm{Chi}^{2}=8.85, \mathrm{df}=7(\mathrm{P}=0.26) \\
\mathrm{I}^{2}=21 \%\end{array}$ & $Z=0.72(P=0.47)$ \\
\hline 4 & $\begin{array}{l}\text { Hypertension + vs. Hyperten- } \\
\text { sion- }\end{array}$ & 8 & 1578 & Fix & $1.07[0.80,1.42]$ & $\begin{array}{l}C h i^{2}=5.33, d f=7(P=0.62) \\
I^{2}=0 \%\end{array}$ & $Z=0.43(P=0.66)$ \\
\hline 5 & $\begin{array}{l}\text { Hyperlipidemia+ } \\
\text { vs. Hyperlipidemia- }\end{array}$ & 8 & 1572 & Fix & $1.13[0.80,1.60]$ & $\begin{array}{l}C \mathrm{Chi}^{2}=3.67, \mathrm{df}=7(\mathrm{P}=0.82) \\
\mathrm{I}^{2}=0 \%\end{array}$ & $Z=0.70(P=0.48)$ \\
\hline 6 & Diabetes + vs. Diabetes- & 8 & 1578 & Fix & $1.26[0.98,1.62]$ & $\begin{array}{l}\mathrm{Chi}^{2}=12.07, \mathrm{df}=7(\mathrm{P}=0.10) \\
\mathrm{I}^{2}=42 \%\end{array}$ & $Z=1.83(P=0.07)$ \\
\hline 7 & Depression+vs. Depression- & 2 & 389 & Fix & $1.36[0.78,2.39]$ & $\begin{array}{l}\mathrm{Chi}^{2}=0.06, \mathrm{df}=1(\mathrm{P}=0.80) \\
\mathrm{I}^{2}=0 \%\end{array}$ & $Z=1.09(P=0.28)$ \\
\hline 8 & Post Ml+ vs. Post Ml- & 5 & 1345 & Fix & $1.29[1.00,1.66]$ & $\begin{array}{l}\mathrm{Chi}^{2}=1.85, \mathrm{df}=4(\mathrm{P}=0.76) \\
\mathrm{I}^{2}=0 \%\end{array}$ & $Z=1.95(P=0.05)$ \\
\hline 9 & PTCA+ vs. PTCA- & 3 & 1128 & Fix & $0.88[0.67,1.16]$ & $\begin{array}{l}C h i^{2}=0.53, d f=2(P=0.77) \\
I^{2}=0 \%\end{array}$ & $Z=0.90(P=0.37)$ \\
\hline 10 & $C A B G+v s . C A B G-$ & 3 & 1128 & Random & $1.59[1.00,2.52]$ & $\begin{array}{l}\operatorname{Tau}^{2}=0.10 ; C h i^{2}=5.18, d f=2 \\
(P=0.07) ; I^{2}=61 \%\end{array}$ & $Z=1.98(P=0.05)$ \\
\hline 11 & Aspirn+ vs. Aspirin- & 5 & 1279 & Fix & $0.93[0.65,1.34]$ & $\begin{array}{l}C h i^{2}=0.34, d f=4(P=0.99) \\
I^{2}=0 \%\end{array}$ & $Z=0.36(P=0.72)$ \\
\hline 12 & $\begin{array}{l}\text { Other antiplatelet agent+ } \\
\text { \& Other antiplatelet agent- }\end{array}$ & 3 & 1128 & Fix & $1.21[0.91,1.61]$ & $\begin{array}{l}C h i^{2}=1.94, d f=2(P=0.38) \\
I^{2}=0 \%\end{array}$ & $Z=1.29(P=0.20)$ \\
\hline 13 & $A C E I+v s . A C E I-$ & 5 & 1279 & Fix & $1.13[0.87,1.46]$ & $\begin{array}{l}C h i^{2}=2.12, d f=4(P=0.71) \\
I^{2}=0 \%\end{array}$ & $Z=0.92(P=0.36)$ \\
\hline 14 & $A R B+v s . A R B-$ & 2 & 967 & Random & $1.22[0.53,2.82]$ & $\begin{array}{l}\mathrm{Tau}^{2}=0.26 ; \mathrm{Chi}^{2}=3.53, \mathrm{df}=1 \\
\quad(P=0.06) ; I^{2}=72 \%\end{array}$ & $Z=0.46(P=0.64)$ \\
\hline 15 & Beta-block+vs. Beta-block- & 6 & 1417 & Fix & $1.05[0.78,1.41]$ & $\begin{array}{l}\mathrm{Chi}^{2}=2.50, \mathrm{df}=5(\mathrm{P}=0.78) \\
\mathrm{I}^{2}=0 \%\end{array}$ & $Z=0.32(P=0.75)$ \\
\hline 16 & $\mathrm{CCB}+$ vs. $\mathrm{CCB}-$ & 4 & 674 & Fix & $0.84[0.58,1.22]$ & $\begin{array}{l}\text { Chi }^{2}=0.78, d f=3(P=0.85) \\
I^{2}=0 \%\end{array}$ & $Z=0.92(P=0.36)$ \\
\hline 17 & Statin+ vs. Statin- & 6 & 1417 & Fix & $1.18[0.80,1.75]$ & $\begin{array}{l}\mathrm{Chi}^{2}=4.30, \mathrm{df}=5(P=0.51) \\
I^{2}=0 \%\end{array}$ & $Z=0.83(P=0.40)$ \\
\hline
\end{tabular}

$A C E l$ angiotensin converting enzyme inhibitor, $A R B$ angiotensin receptor block, $C C B$ calcium-channel blocker, $M I$ myocardial ischemia

1.0187, SE: 0.0282, P: 0.512). Therefore, different types of mental stress might contribute to the prevalence of MSIMI.

Multivariate meta-regression was performed including mental stress, diagnostic technique, and diagnostic criteria. No significant difference was found: mental stress (exp(b): 1.0433, SE: 0.0260, P: 0.108), diagnostic techniques $(\exp (\mathrm{b}): 1.0145, \mathrm{SE}: 0.0239, \mathrm{P}: 0.551)$, diagnostic measurements $(\exp (\mathrm{b}): 1.0263$, SE: $0.0250, \mathrm{P}: 0.302)$ (Table 5).

Our results indicated that different types of mental stress might influence the prevalence of MSIMI in CAD patients.

\section{Comparisons of different diagnostic techniques}

In all twenty selected articles, there were four articles indicating that different diagnostic techniques might lead to different prevalence of MSIMI in the same population. Two articles compared SPECT and PAT (peripheral arterial tonometry) which was not recognized as a standard criterion. In Burg's study, the prevalence of MSIMI was $32.35 \%$ by SPECT, and $42.65 \%$ by PAT, while only $19.12 \%$ by both. The area under the curve (AUC) was 0.613 (SE, 0.065, onesided $\mathrm{P}=0.04)$. In Hassan's study, when comparing SPECT and PAT, the area under the curve (AUC) was 0.59 (95\% CI $0.48-0.69, \mathrm{P}=0.116$ ). In addition, Carels' study showed that the prevalence of MSIMI was $33.09 \%$ by RNV, and $44.12 \%$ by ambulatory ECG, while only $19.2 \%$ by both. Krantz's study showed that the prevalence of MSIMI was $55.7 \%$ by RNV, and $57 \%$ by echocardiography (Table 6 ). 


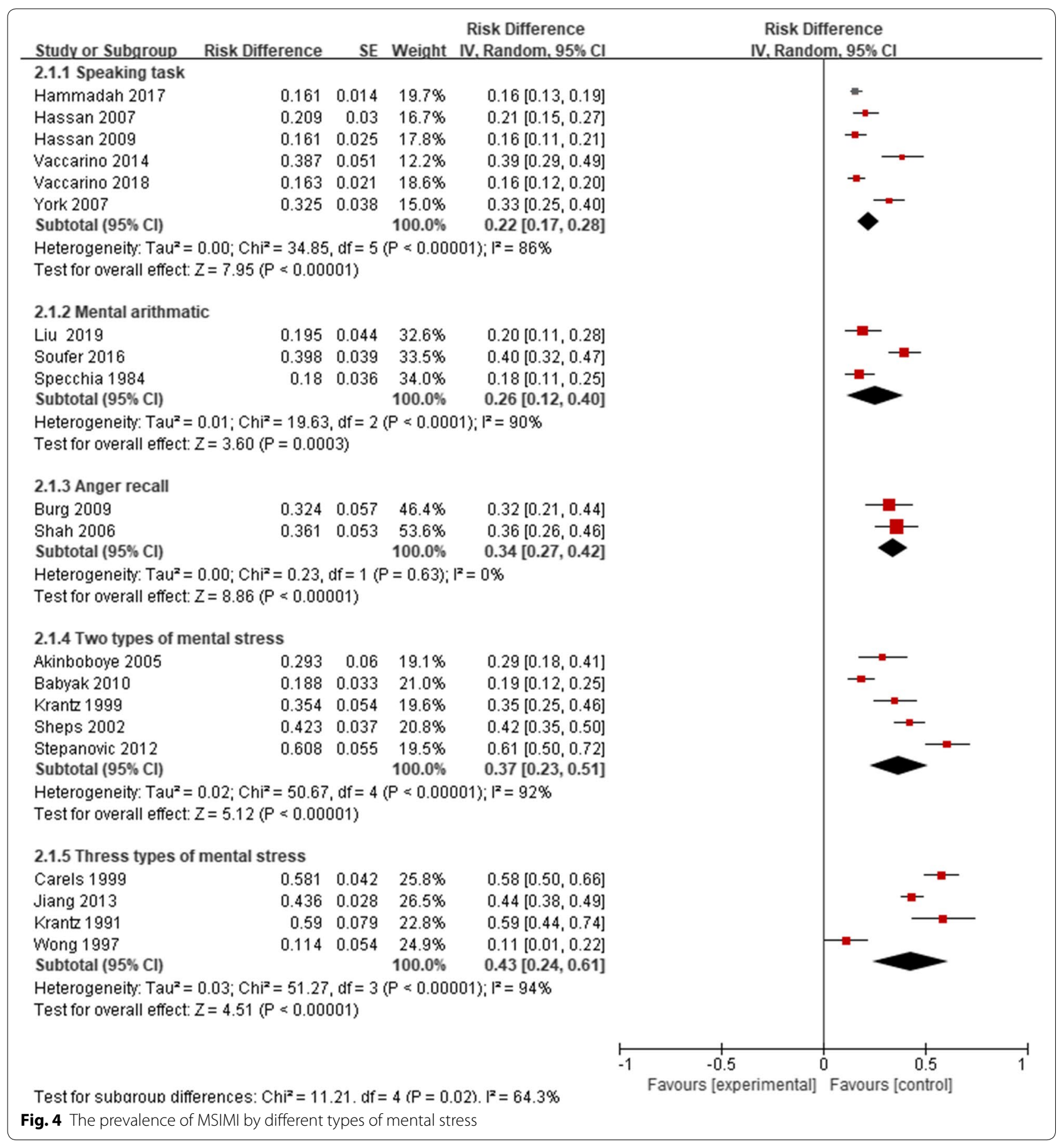

\section{Sensitivity analysis}

We performed sensitivity analysis by Stata 12.0, excluding a single study each time to detect the influence of individual dataset on pooled ESs. The results demonstrated that no significant change was found after omitting any of the study (Fig. 7).

\section{Publication bias}

Publication bias was detected by funnel plot and modified Begg' test. The funnel plot was symmetric and the Begg' test presented no significant publication bias in this meta-analysis $(\mathrm{Z}=1.69, \mathrm{P}>0.05)$ (Fig. 8). 


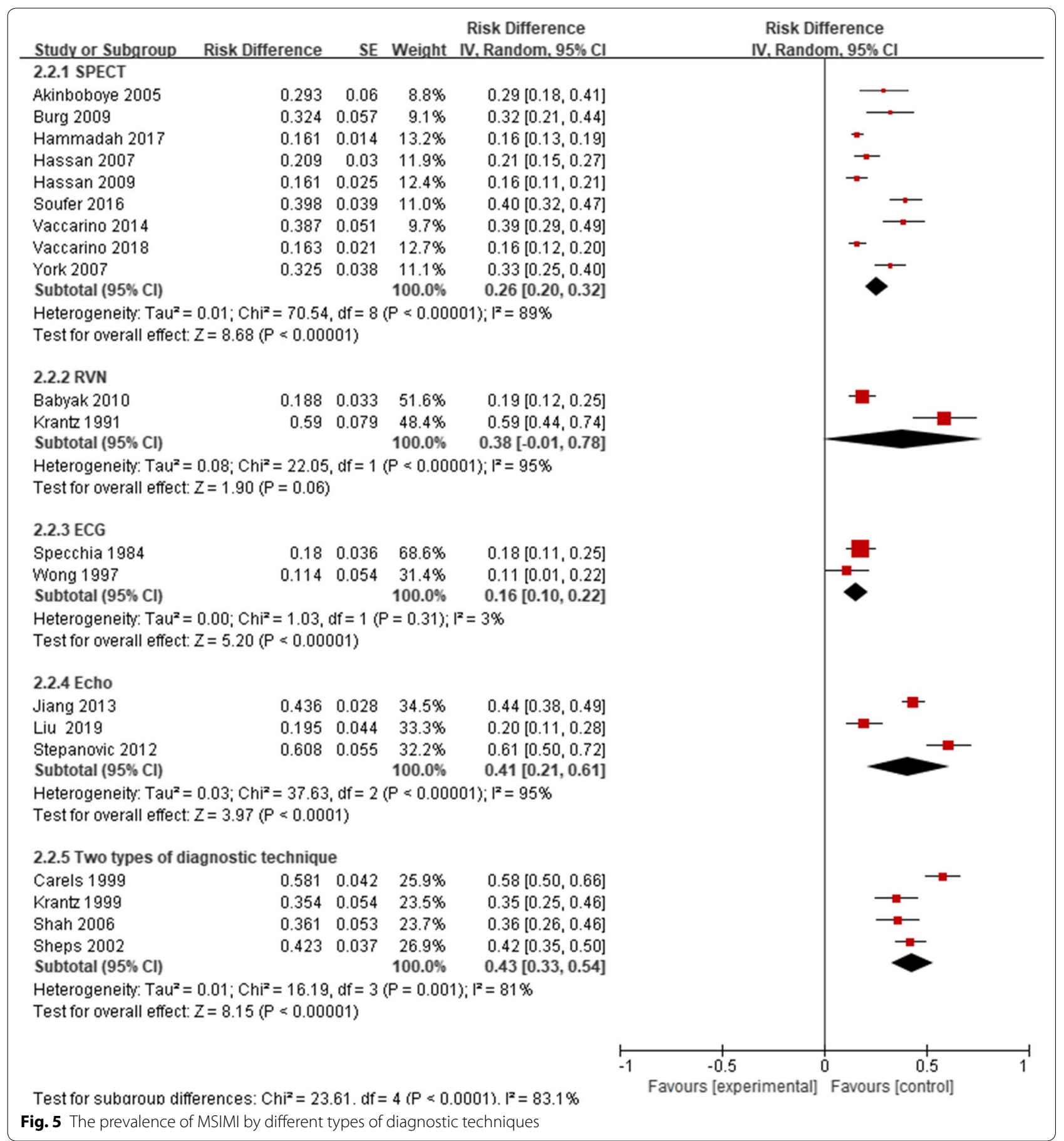

\section{Discussion}

In this meta-analysis, the pooled estimated prevalence of MSIMI in CAD patients was as high as $32 \%$. Consequently, it attracted our attentions to summarize the related factors and diagnostic methods of MSIMI in CAD patients to better understand the MSIMI assessment. To the best of our knowledge, this is the first meta-analysis concentrated on this topic.

\section{Associated factors of MSIMI}

Subgroups analyses elucidated that CAD patients with history of diabetes, or post MI, or CABG might be 


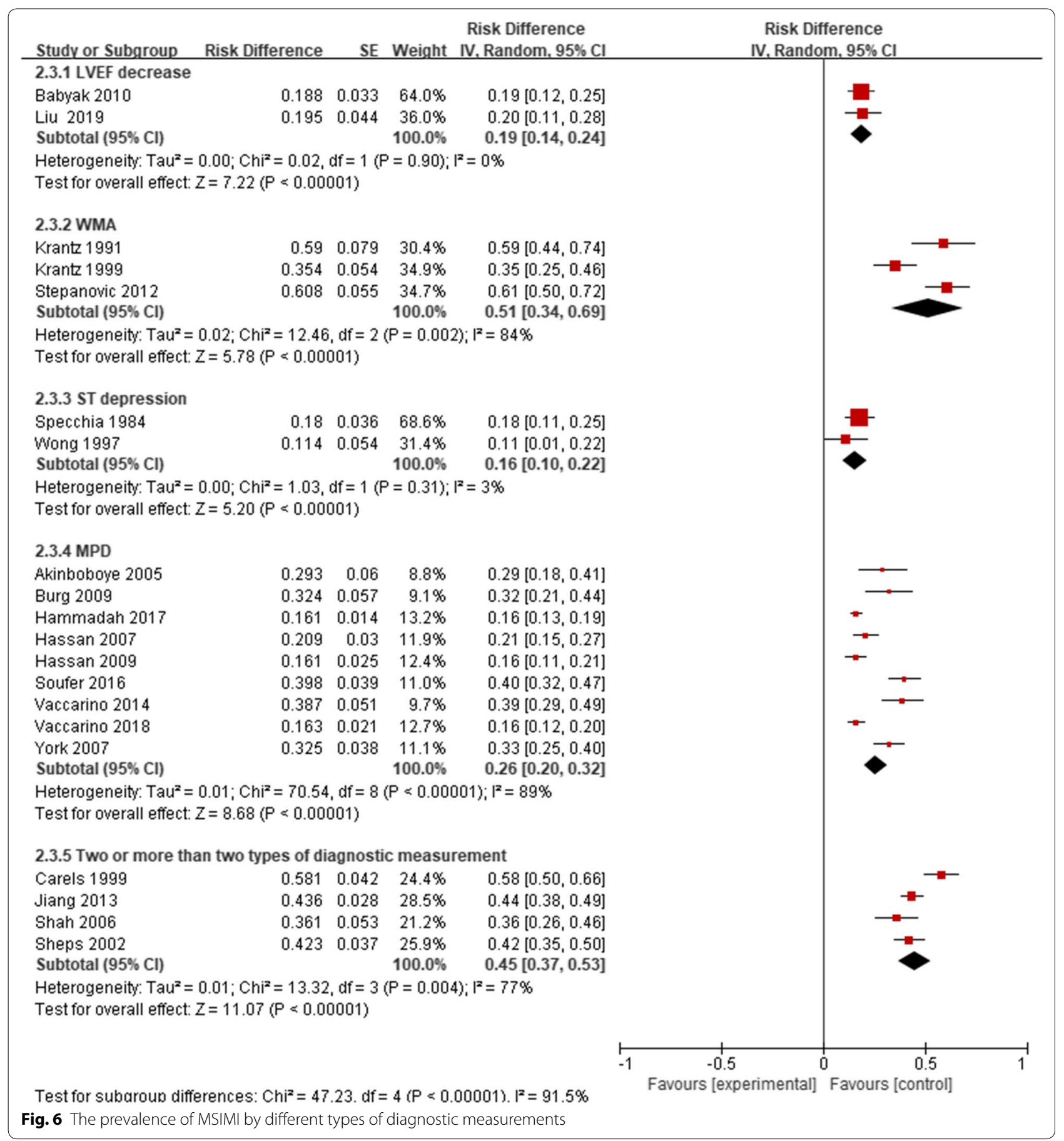

associated with a higher risk of MSIMI, though the statistical analysis was not significant enough. Diabetes is considered as a risk factor of CAD, due to the dysfunction of micro- and macro- vascular damaged by hyperglycemia [38] via inflammation pathway. The sudden mental stress results in the lack of blood flow and oxygen, and thus causes myocardial ischemia [9]. Patients with diabetes, or post $\mathrm{MI}$, or $\mathrm{CABG}$, have worse cardiac conditions because of existing cardiac cell damage and microvascular dysfunction. Therefore, they are more vulnerable to myocardial ischemia when mental stress occurs.

Our results indicated that there was no significant difference of developing MSIMI between females and males, and between patients with depression and without. The 
Table 5 Univariate and multivariate meta-regression analyses of potential sources of heterogeneity

\begin{tabular}{|c|c|c|c|c|c|c|c|c|}
\hline Heterogeneity Factors & $\exp (b)$ & SE & $\mathrm{t}$ & $\mathbf{P}$ & $95 \% \mathrm{Cl}$ & $\operatorname{tau}^{2}$ & I-squared_res & Adj R-squared \\
\hline \multicolumn{9}{|l|}{ Univariate } \\
\hline Publication year & 0.9977 & 0.0038 & -0.61 & 0.549 & $0.9896,1.0058$ & 0.0216 & $93.69 \%$ & $-4.07 \%$ \\
\hline Sample size & 0.9996 & 0.0002 & -1.29 & 0.215 & $0.9992,1.0002$ & 0.0200 & $92.70 \%$ & $3.56 \%$ \\
\hline Country & 0.9433 & 0.0815 & -0.68 & 0.508 & $0.7867,1.1311$ & 0.0214 & $94.23 \%$ & $-3.41 \%$ \\
\hline Mental stress & 1.0508 & 0.0201 & 2.59 & 0.018 & $1.0094,1.0938$ & 0.0151 & $88.73 \%$ & $26.96 \%$ \\
\hline Diagnostic techniques & 1.0395 & 0.0200 & 2.01 & 0.060 & $0.9983,1.0825$ & 0.017 & $89.82 \%$ & $17.71 \%$ \\
\hline Diagnostic measurements & 1.0187 & 0.0282 & 0.67 & 0.512 & $0.9611,1.0797$ & 0.0212 & $93.95 \%$ & $-2.46 \%$ \\
\hline Multivariate & & & & & & 0.0151 & $87.62 \%$ & $26.97 \%$ \\
\hline Mental stress & 1.0433 & 0.0260 & 1.70 & 0.108 & $0.9896,1.0999$ & & & \\
\hline Diagnostic techniques & 1.0145 & 0.0239 & 0.61 & 0.551 & $0.9650,1.0665$ & & & \\
\hline Diagnostic measurements & 1.0263 & 0.0250 & 1.07 & 0.302 & $0.9747,1.0807$ & & & \\
\hline
\end{tabular}

results in this meta-analysis were different from some individual studies.

Vaccarino et al. [35] elucidated that young women with CHD were more likely to develop MSIMI, which was almost fourfold higher than men. Another study of Vaccarino reported similar conclusions that mechanisms in MSIMI could be different in females and males, and the higher morbidity of MSIMI in females might be related with the microcirculatory dysfunction. Samad et al. [39] suggested that the higher morbidity of MSIMI in females might be associated with platelet activity. To our surprise, our results in the present study did not suggest sex as a significant risk factor. This inconsistency might be due to: (1) the different samples and proportion of females and males in each study; (2) the studies were from different regions. More original researches should be done to further study the relationship between sex and MSIMI.

Depression is an independent risk factor of cardiovascular diseases [40]. Jiang et al. [7] suggested that patients with mild to moderate depressive symptoms were at higher risk of MSIMI. In this research, depression was assessed by Center for Epidemiological StudiesDepression scale (CES-D). However, only four articles mentioning depression were included in the present meta-analysis, and no significant importance was found in depression as a risk factor for MSIMI.

In addition, anger [5], sever left ventricular dysfunction, and anxiety [6] have been considered as severe factors in MSIMI, but the evidence is not enough.

\section{Diagnostic methods of MSIMI}

We found significant differences in MSIMI prevalence detected by different mental stress, diagnostic techniques and diagnostic measurement. Univariate metaregression elucidated the potential link between types of mental stress and MSIMI. We postulated some potential reasons for this association. First, the activation of different signal pathway may lead to different consequences. The mechanism of MSIMI involves the strong interaction between heart and the brain. Mental stress can activate hypothalamic pituitary adrenocortical axis, sympathetic nervous system, adrenomedullar hormonal system, and parasympathemic nervous system via releasing different hormones or neurotransmitters which can have different impact. Second, individual differences may play an important role. In Table 1, we described the types of mental stress in all the included studies. The common types involve mental arithmetic, anger call, public speech, mirror trace, Stroop color-word test et al. We found that the prevalence of MSIMI induced by one type of mental stress was 22-34\%, two types of mental stress was $37 \%$, and three types yield $43 \%$ (Table 3 ). According to our own clinical observations, trace mirror seemed to be a pleasure rather than emotional stress for those who are good at designing or drawing, while mental arithmetic could be a serious stress to them for most of them are afraid of mathematics; vise verse for those who are skilled at mental arithmetic. The phenomenon implied that we should consider individual differences in the consequence caused by different types of mental stress task, which is consistent with Bremner et al's study. Bremner [41] conducted a study with the intent of revealing the association between brain and MSIMI. It was found that mental arithmetic was associated with left insula activation, while public speaking was associated with right pre/ post-central gyrus and middle temporal gyrus activation. In the context of MSIMI, different types of mental stress might active or deactivate different brain regions, which would promote or inhibit cardiac responses. Therefore, we suggest that researchers should consider individual differences in different types of mental stress task while assessing MSIMI, and make a standard together. In our 


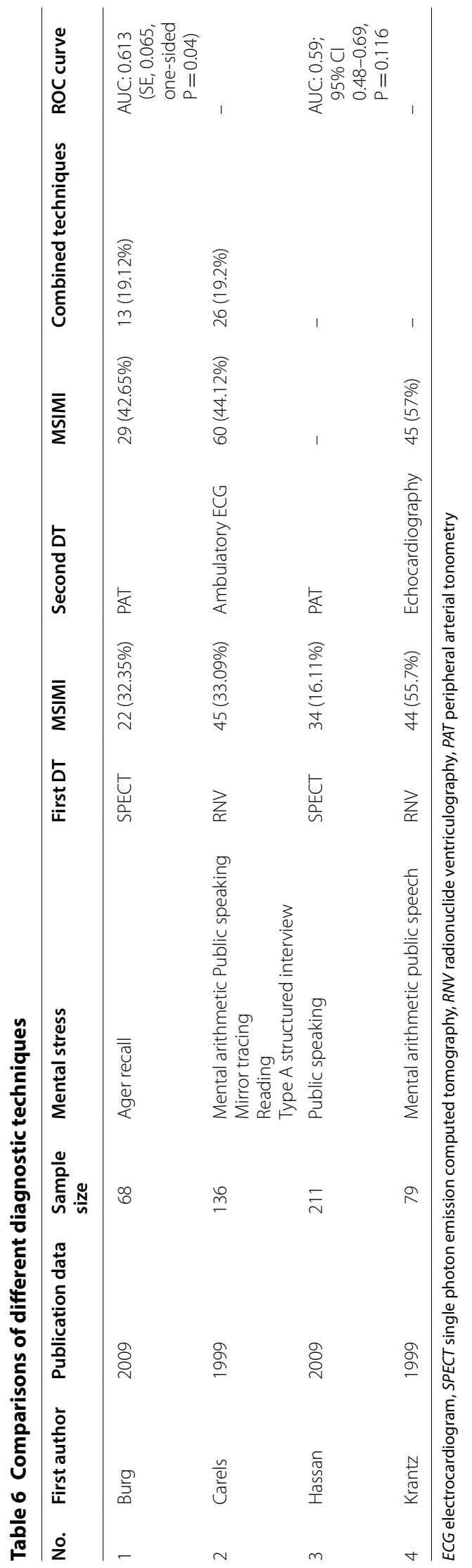



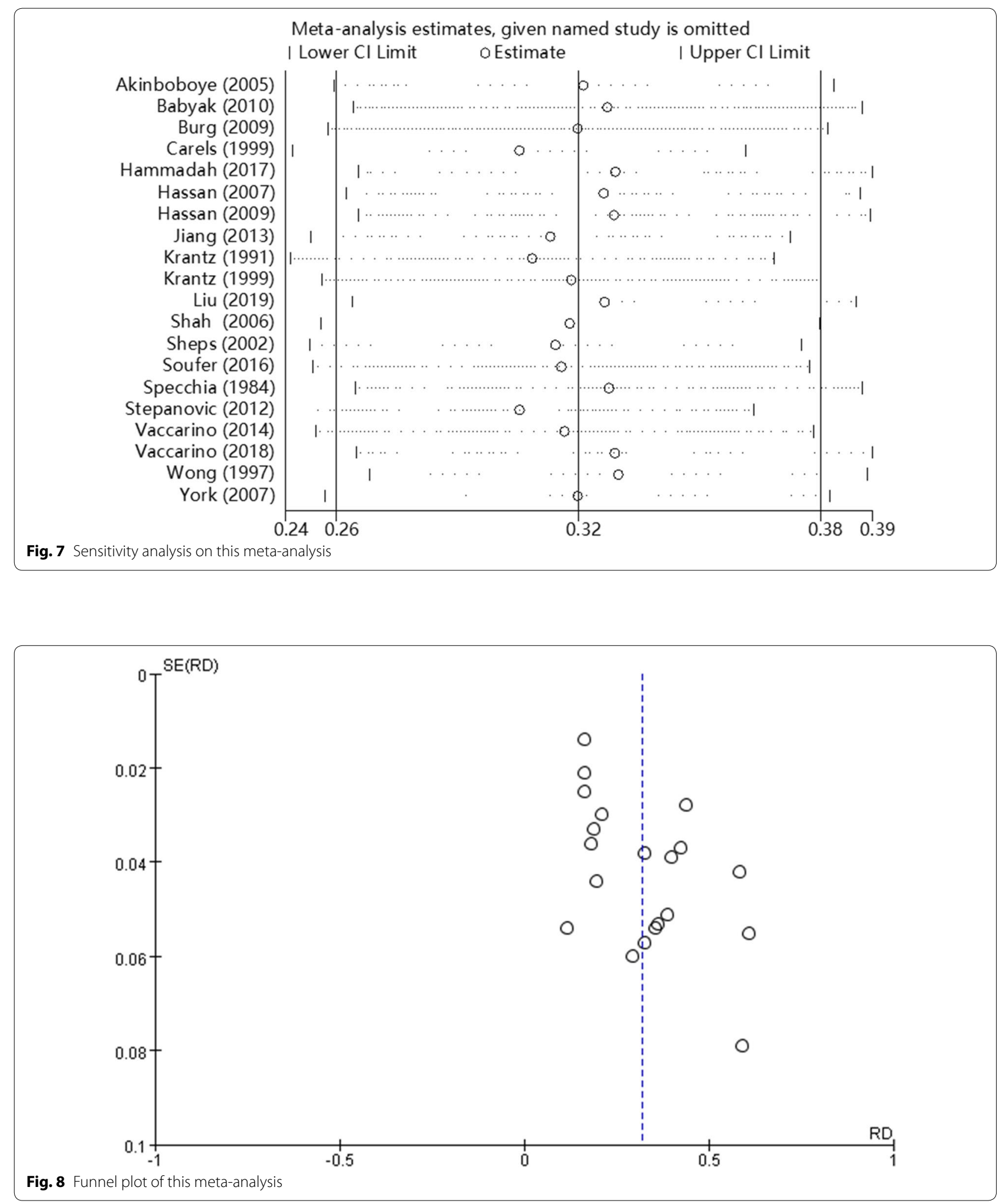
opinion, two different types of mental stress tasks would be better to diagnose MSIMI for the reason that one type might not be eligible to provoke MSIMI, while more than two types might be time and economic consuming.

In addition, we took diagnostic techniques as a pivotal factor in diagnosing MSIMI. As Table 3 showed that the prevalence of MSIMI diagnosed by SPECT was 26\%, ECG yielded $16 \%$, while echocardiography yielded $41 \%$, RNV yields $38 \%$. SPECT is a direct way to observe myocardial ischemia via myocardial perfusion defects, demonstrating its vital role in diagnosing MSIMI. Good reproducibility of SPECT has also been identified [42]. However, some patients with MSIMI assessed by echocardiography might be missed.

ECG is a convenient technique, but it is been proved not sensitive enough for MSIMI [36]. Jiang et al. [25] investigated both ECG and echocardiography in distinguishing MSIMI, while no myocardial ischemia was discovered by ECG. Therefore, the false negative of ECG presented low prevalence of MSIMI.

Echocardiography is economical and practical in clinical practice, which could detect LVEF response and wall motion during mental stress simultaneously. Though LVEF decrease could result from myocardial ischemia induced by mental stress and also be consistent with SPECT [43], LVEF response is also influenced by hemodynamics and the basic left ventricular function [18]. Therefore, echocardiography is likely to generate false positive results.

Peripheral arterial tonometry (PAT) is applied to assess microcirculation dysfunction, which is expected to detect myocardial ischemia induced by mental stress. CAD patients with MSIMI have lower PAT ratio according to the studies comparing SPECT and PAT. Some researchers suggested that PAT might have similar detection efficiency compared to SPECT and RNV [20,44], and more researches remain to further explore the potential role of PAT in detecting MSIMI and make it standardized.

Additionally, increasing researches have been focused on biomarkers that are convenient to achieve and assess, such as neurotransmitters (e.g. epinephrine, norepinephrine [45]), blood coagulation factors (e.g. fibrinogen [46]), cardiac biomarkers (e.g. cTnI [11], cTnT [46]), and inflammatory factors (e.g. IL-6 [47], CRP [29]). These biomarkers are considered to the mechanisms of MSIMI. Consequently, there is bright future in discovering biomarkers for developing economic diagnostic methods of MSIMI.

\section{Conclusions}

In conclusion, the pooled prevalence of MSIMI in CAD patients is $32 \%$. The present meta-analysis implicates that patients with diabetes, or post MI or CABG are more vulnerable to develop MSIMI and different types of mental stress and diagnostic techniques might influence the prevalence of MSIMI. Therefore, it is necessary to formulate a standard diagnostic method for MSIMI, which should be adequate, assessable, and affordable all around world.

\section{Abbreviations \\ MSIMI: Mental stress induced myocardial ischemia; CAD: Coronary artery diseases; MI: Myocardial infarction; LVEF: Left ventricular ejection fraction; WMA: Wall motion abnormality; ECG: Electrocardiogram; RNV: Radionuclide ventriculography; PTCA: Percutaneous coronary angioplasty; CABG: Coronary artery bypass graft; ACEl: Angiotensin converting enzyme inhibitor; ARB: Angiotensin receptor block; CCB: Calcium-channel blocker; CVD: Cardiovas- cular diseases; SPECT: Single photonemission computed tomography; MPD: Myocardial perfusion defects; PAT: Peripheral arterial tonometry; CNKI: China National Knowledge Infrastructure; NOS: Newcastle-Ottawa Scale; CES-D: Center for Epidemiological Studies-Depression scale; HPA: Hypothalamic pituitary adrenal.}

\section{Acknowledgements \\ Sincere thanks to Prof. Xiantao Zeng for directing this meat-analysis.}

\section{Authors' contributions}

ML designed the research protocol and revised the manuscript; YB conducted the data analysis and revised the manuscript; LZ wrote the manuscript; $Y Z$ revised the manuscript; $X W, S T$ and $W X$ performed data selection and extracted data. All authors read and approved the final manuscript.

\section{Funding}

National Academy of Innovation Strategy (2019ys 1-4-4-8) and China Women's Development Foundation (2019300) support this work.

\section{Availability of data and materials}

Data and materials will be provided to those who are interested in this metaanalysis by the correspondence.

Ethics approval and consent to participate

Not applicable.

Consent for publication

All the authors agree with the publication of this meta-analysis.

Competing interests

All the authors declared no competing interests in this manuscript.

\section{Author details \\ ${ }^{1}$ Cardiology Department, Beijing Anzhen Hospital, Capital Medical University, No. 2 Anzhen Road, Chaoyang District, Beijing 100029, China. ${ }^{2}$ National Insti- tute on Drug Dependence, Peking University, No. 38, Xueyuan Road, Haidian District, Beijing 10091, China. ${ }^{3}$ Department of Anatomy and Neurobiology, Boston University, Boston, USA. ${ }^{4}$ School of Basic Medical Sciences, Henan University, Kaifeng, China.}

Received: 25 March 2020 Accepted: 16 May 2020

Published online: 29 May 2020 


\section{References}

1. Neylon A, Canniffe C, Anand S, Kreatsoulas C, Blake GJ, Sugrue D, McGorrian C. A global perspective on psychosocial risk factors for cardiovascular disease. Prog Cardiovasc Dis. 2013;55:574-81.

2. Hu SSGR, Liu LS, Zhu ML, Wang W, Wang YJ, Wu ZS, Li HJ, Gu DF, Yang YJ, Zheng Z, Chen WW, on behalf of the Writing Committee of the Report on Cardiovascular Diseases in China. Summary of the 2018 report on cardiovascular diseases in China. Chin Circul J. 2019;34:209.

3. Almourani RCB, Patel R, Kurukulasuriya LR, Sowers J. Diabetes and cardiovascular disease: an update. Curr Diab Rep. 2019;19:161.

4. Albus C, Waller C, Fritzsche K, Gunold H, Haass M, Hamann B, Kindermann I, Kollner V, Leithauser B, Marx N, et al. Significance of psychosocial factors in cardiology: update 2018: position paper of the German Cardiac Society. Clin Res Cardiol. 2019:108:1175-96.

5. Williams RB. Anger and mental stress-induced myocardial ischemia: mechanisms and clinical implications. Am Heart J. 2015;169:4-5.

6. Allgulander C. Anxiety as a risk factor in cardiovascular disease. Curr Opin Psychiatry. 2016;29:13-7.

7. Jiang W, Babyak MA, Rozanski A, Sherwood A, O'Connor CM, Waugh RA, Coleman RE, Hanson MW, Morris JJ, Blumenthal JA. Depression and increased myocardial ischemic activity in patients with ischemic heart disease. Am Heart J. 2003;146:55-61.

8. Jiang W, Babyak M, Krantz DS, Waugh RA, Coleman RE, Hanson MM, Frid DJ, McNulty S, Morris JJ, Oconnor CM, Blumenthal JA. Mental stress-induced myocardial ischemia and cardiac events. J Am Med Assoc. 1996;275:1651-6.

9. Soufer R, Arrighi JA, Burg MM. Brain, behavior, mental stress, and the neurocardiac interaction. J Nucl Cardiol. 2002;9:650-62.

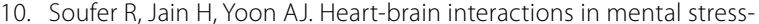
induced myocardial ischemia. Curr Cardiol Rep. 2009;11:133-40.

11. Hammadah M, Al Mheid I, Wilmot $K$, et al. Association between highsensitivity cardiac troponin levels and myocardial ischemia during mental stress and conventional stress. JACC Cardiovasc Imaging. 2018;11:603-11.

12. Steele E, Bialocerkowski A, Grimmer K. The postural effects of load carriage on young people-a systematic review. BMC Musculoskelet Disord. 2003;4:12.

13. Ma LL, Wang YY, Yang ZH, Huang D, Weng H, Zeng XT. Methodological quality (risk of bias) assessment tools for primary and secondary medical studies: what are they and which is better? Mil Med Res. 2020;7:7.

14. DerSimonian R, Laird N. Meta-analysis in clinical trials. Control Clin Trials. 1986:7:177-88.

15. Melsen WG, Bootsma MC, Rovers MM, Bonten MJ. The effects of clinical and statistical heterogeneity on the predictive values of results from meta-analyses. Clin Microbiol Infect. 2014;20:123-9.

16. Tobias A. Assessing the influence of a single study in meta-analysis. Stata Tech Bull. 1999:8:47.

17. Begg CB, Mazumdar M. Operating characteristics of a rank correlation test for publication bias. Biometrics. 1994;50:1088-101.

18. Akinboboye O, Krantz DS, Kop WJ, Schwartz SD, Levine J, Del Negro A Karasik P, Berman DS, O'Callahan M, Ngai K, Gottdiener JS. Comparison of mental stress-induced myocardial ischemia in coronary artery disease patients with versus without left ventricular dysfunction. Am J Cardiol. 2005;95:322-6.

19. Babyak MA, Blumenthal JA, Hinderliter A, Hoffman B, Waugh RA, Coleman RE, Sherwood A. Prognosis after change in left ventricular ejection fraction during mental stress testing in patients with stable coronary artery disease. Am J Cardiol. 2010;105:25-8.

20. Burg MM, Graeber B, Vashist A, Collins D, Earley C, Liu J, Lampert R, Soufer R. Noninvasive detection of risk for emotion-provoked myocardial ischemia. Psychosom Med. 2009;71:14-20.

21. Carels RA, Sherwood A, Babyak M, Gullette EC, Coleman RE, Waugh R, Jiang W, Blumenthal JA. Emotional responsivity and transient myocardial ischemia. J Consult Clin Psychol. 1999:67:605-10.

22. Hammadah M, Al Mheid I, Wilmot K, Ramadan R, Shah AJ, Sun Y, Pearce B, Garcia EV, Kutner M, Bremner JD, et al. The mental stress ischemia prognosis study: objectives, study design, and prevalence of inducible ischemia. Psychosom Med. 2017:79:311-7.

23. Hassan M, York KM, Li H, Li Q, Sheps DS. Mental stress-induced myocardial ischemia in coronary artery disease patients with left ventricular dysfunction. J Nucl Cardiol. 2007;14:308-13.
24. Hassan M, York KM, Li H, Li Q, Lucey DG, Fillingim RB, Sheps DS. Usefulness of peripheral arterial tonometry in the detection of mental stressinduced myocardial ischemia. Clin Cardiol. 2009;32:E1-6.

25. Jiang W, Samad Z, Boyle S, Becker RC, Williams R, Kuhn C, Ortel TL, Rogers J, Kuchibhatla M, O'Connor C, Velazquez EJ. Prevalence and clinical characteristics of mental stress-induced myocardial ischemia in patients with coronary heart disease. J Am Coll Cardiol. 2013;61:714-22.

26. Krantz DS, Helmers KF, Bairey CN, Nebel LE, Hedges SM, Rozanski A. Cardiovascular reactivity and mental stress-induced myocardial ischemia in patients with coronary artery disease. Psychosom Med. 1991:53:1-12.

27. Krantz DS, Santiago HT, Kop WJ, Bairey Merz CN, Rozanski A, Gottdiener JS. Prognostic value of mental stress testing in coronary artery disease. Am J Cardiol. 1999;84:1292-7.

28. Liu MY, Yang Y, Zhang LJ, Pu LH, He DF, Liu JY, Hafeez A, Ding YC, Ma $H$, Geng QS. Potential predictors for mental stress-induced myocardial ischemia in patients with coronary artery disease. Chin Med J (Engl). 2019;132:1390-9.

29. Shah R, Burg MM, Vashist A, Collins D, Liu J, Jadbabaie F, Graeber B, Earley C, Lampert R, Soufer R. C-reactive protein and vulnerability to mental stress-induced myocardial ischemia. Mol Med. 2006;12:269-74.

30. Sheps DS, McMahon RP, Becker L, Carney RM, Freedland KE, Cohen JD, Sheffield D, Goldberg AD, Ketterer MW, Pepine CJ, et al. Mental stressinduced ischemia and all-cause mortality in patients with coronary artery disease: results from the Psychophysiological Investigations of Myocardial Ischemia study. Circulation. 2002;105:1780-4.

31. Soufer R, Fernandez AB, Meadows J, Collins D, Burg MM. Body mass index and risk for mental stress induced ischemia in coronary artery disease. Mol Med. 2016;22:286-91.

32. Specchia G, De Servi S, Falcone C. Mental arithmetic stress testing in patients with coronary artery disease. Am Heart J. 1984;108:56-63.

33. Stepanovic J, Ostojic M, Beleslin B, Vukovic O, Dikic AD, Giga V, Nedeljkovic I, Nedeljkovic M, Stojkovic S, Vukcevic V, et al. Mental stressinduced ischemia in patients with coronary artery disease: echocardiographic characteristics and relation to exercise-induced ischemia: erratum. Psychosom Med. 2012;74:989.

34. Vaccarino V, Shah AJ, Rooks C, Ibeanu I, Nye JA, Pimple P, Salerno A D'Marco L, Karohl C, Bremner JD, Raggi P. Sex differences in mental stress-induced myocardial ischemia in young survivors of an acute myocardial infarction. Psychosom Med. 2014;76:171-80.

35. Vaccarino V, Sullivan S, Hammadah M, Wilmot K, Al Mheid I, Ramadan R, Elon L, Pimple PM, Garcia EV, Nye J, et al. Mental stress-induced-myocardial ischemia in young patients with recent myocardial infarction: sex differences and mechanisms. Circulation. 2018;137:794-805.

36. Wong CK, BenFreedman S. Usefulness of laboratory mental stress test in patients with stable coronary artery disease. Clin Cardiol. 1997;20:367-71.

37. York KM, Hassan M, Li Q, Li H, Fillingim RB, Lucey D, Bestland M, Sheps DS. Do men and women differ on measures of mental stress-induced ischemia? Psychosom Med. 2007;69:918-22.

38. Fiorentino TV, Prioletta A, Zuo P, Folli F. Hyperglycemia-induced oxidative stress and its role in diabetes mellitus related cardiovascular diseases. Curr Pharm Des. 2013;19:5695-703.

39. Samad Z, Boyle S, Ersboll M, Vora AN, Zhang Y, Becker RC, Williams R, Kuhn C, Ortel TL, Rogers JG, et al. Sex differences in platelet reactivity and cardiovascular and psychological response to mental stress in patients with stable ischemic heart disease: insights from the REMIT study. J Am Coll Cardiol. 2014;64:1669-78.

40. Sharovsky LLRB, Franchini Ramires JA. Perception of depressive symptoms in patients after myocardial infarction: qualitative study. Heart Mind. 2017:1:107-11.

41. Bremner JD, Campanella C, Khan Z, Shah M, Hammadah M, Wilmot K, Al Mheid I, Lima BB, Garcia EV, Nye J, et al. Brain correlates of mental stress-induced myocardial ischemia. Psychosom Med. 2018;80:515-25.

42. Kim CK, Bartholomew BA, Mastin ST, Taasan VC, Carson KM, Sheps DS. Detection and reproducibility of mental stress-induced myocardial ischemia with Tc-99 $\mathrm{m}$ sestamibi SPECT in normal and coronary artery disease populations. J Nucl Cardiol. 2003;10(1):56-62.

43. Kuroda T, Kuwabara Y, Watanabe S, Nakaya J, Hasegawa R, Shikama T, Matsuno K, Mikami Y, Fujii K, Saito T, Masuda Y. Effect of mental stress 
on left ventricular ejection fraction and its relationship to the severity of coronary artery disease. Eur J Nucl Med. 2000;27:1760-7.

44. Goor DA, Sheffy J, Schnall RP, Arditti A, Caspi A, Bragdon EE, Sheps DS Peripheral arterial tonometry: a diagnostic method for detection of myocardial ischemia induced during mental stress tests: a pilot study Clin Cardiol. 2004;27:137-41.

45. Dakak N, Quyyumi AA, Eisenhofer G, Goldstein DS, Cannon IRO. Sympathetically mediated effects of mental stress on the cardiac microcirculation of patients with coronary artery disease. Am J Cardiol. 1995;76:125-30.

46. Lazzarino Al, Hamer M, Gaze D, Collinson P, Rumley A, Lowe G, Steptoe A. The association between fibrinogen reactivity to mental stress and high-sensitivity cardiac troponin T in healthy adults. Psychoneuroendocrinology. 2015;59:37-48.

47. Hammadah M, Sullivan S, Pearce B, Al Mheid I, Wilmot K, Ramadan R, Tahhan AS, O'Neal WT, Obideen M, Alkhoder A, et al. Inflammatory response to mental stress and mental stress induced myocardial ischemia. Brain Behav Immun. 2018;68:90-7.

\section{Publisher's Note}

Springer Nature remains neutral with regard to jurisdictional claims in published maps and institutional affiliations.
Ready to submit your research? Choose BMC and benefit from:

- fast, convenient online submission

- thorough peer review by experienced researchers in your field

- rapid publication on acceptance

- support for research data, including large and complex data types

- gold Open Access which fosters wider collaboration and increased citations

- maximum visibility for your research: over $100 \mathrm{M}$ website views per year

At BMC, research is always in progress.

Learn more biomedcentral.com/submissions 